\title{
Q7-branes and their coupling to IIB supergravity
}

\author{
Eric Bergshoeff and Jelle Hartong \\ Centre for Theoretical Physics, University of Groningen, \\ Nijenborgh 4, 9747 AG Groningen, The Netherlands \\ E-mail: e.a.bergshoeff@rug.nl, j.hartong@rug.nl
}

\section{Dmitri Sorokin}

INFN, Sezione di Padova and Dipartimento di Fisica "Galileo Galilei", Università degli Studi di Padova, via F. Marzolo 8, 35131 Padova, Italia, and Departamento de Fisica Teórica, Facultad de Fisica, Universidad de Valencia, C/Dr. Moliner, 50, 46100 Burjassot (Valencia), España E-mail: dmitri.sorokin@pd.infn.it

\begin{abstract}
We show how, by making use of a new basis of the IIB supergravity axiondilaton coset, $\mathrm{SL}(2, \mathbb{R}) / \mathrm{SO}(2)$, 7-branes that belong to different conjugacy classes of the duality group $\mathrm{SL}(2, \mathbb{R})$ naturally couple to IIB supergravity with appropriate source terms characterized by an $\mathrm{SL}(2, \mathbb{R})$ charge matrix $\mathrm{Q}$. The conjugacy classes are determined by the value of the determinant of $\mathrm{Q}$. The $(p, q)$ 7-branes are the branes in the conjugacy class $\operatorname{det} Q=0$. The 7 -branes in the conjugacy class $\operatorname{det} Q>0$ are labelled by three numbers $(p, q, r)$ which parameterize the matrix $Q$ and will be called Q7-branes. We construct the full bosonic Wess-Zumino term for the Q7-branes. In order to realize a gauge invariant coupling of the Q7-brane to the gauge fields of IIB supergravity it is necessary to introduce an $\operatorname{SL}(2, \mathbb{R})$ doublet of two distinct Born-Infeld fields on the Q7-brane world-volume.
\end{abstract}

KEYWORDs: p-branes, D-branes. 


\section{Contents}

1. Introduction 2

2. D7-brane coupling 3

3. Q7-brane coupling 6

3.1 Q7-branes in the $\left(T, \chi^{\prime}\right)$ basis

3.2 Unobservability of the Dirac 8-brane 9

3.3 Field equations and static 7-brane solutions 9

4. Dirac strings and monodromy 11

4.1 Q7-branes in the $(\tau, \bar{\tau})$ basis 11

4.2 Monodromy 12

4.3 Multiple 7-branes 14

5. Conjugacy class dependence of IIB supergravity 15

5.1 Coordinate systems on the scalar manifold 16

5.2 What about $\operatorname{det} Q<0$ ? 18

6. Towards the construction of the Q7-brane world-volume action 19

6.1 The Wess-Zumino term 19

6.2 The Dirac-Born-nfeld part of the Q7-brane action 21

7. Discussion 22

A. Conventions 24

B. Properties of the $\mathrm{SU}(1,1) / \mathrm{U}(1)$ scalar coset 24

G. IIB supergravity 25

C.1 Manifest $\mathrm{SU}(1,1)$ covariant formulation 25

C.2 Introducing 6-forms 26

C.3 Introducing 8-forms 27

C.4 Gauge field transformations 28

C.5 Manifest $\operatorname{SL}(2, \mathbb{R})$ covariant formulation 28 


\section{Introduction}

In [1] 7-brane solutions of IIB supergravity have been investigated with an emphasis on their supersymmetry properties. One of the results of that paper was the observation that the construction of globally well-defined supersymmetric 7-brane solutions from a 10dimensional point of view can be realized provided one introduces a new type of 7-brane.

It has been proposed in [2] that 7-branes are described by a triplet of charges here denoted by $(p, q, r)$. This is in contrast with the common statement that in type IIB superstring theory there are only $(p, q)$ 7-branes. The main argument of [2] in support of this idea was that the RR 8-form transforms as part of a triplet of 8 -forms under $\operatorname{SL}(2, \mathbb{R}) .{ }^{1}$ This observation was also made in [3]. The $(p, q, r)$ charges naturally parameterize an $\mathrm{SL}(2, \mathbb{R})$ algebra valued matrix $Q$

$$
Q=\left(\begin{array}{cc}
r / 2 & p \\
-q & -r / 2
\end{array}\right) .
$$

So an arbitrary $\mathrm{SL}(2, \mathbb{R})$ transformation can be written as $e^{Q}$. In $\llbracket$ it was shown that at least locally one can write down three families of 7 -brane solutions parameterized by the value of $\operatorname{det} Q$. The three families $\operatorname{depend}$ on whether $\operatorname{det} Q<0, \operatorname{det} Q=0 \operatorname{or} \operatorname{det} Q>0$. The D7-brane corresponds to $p=1$ and $q=r=0$, i.e. it has $\operatorname{det} Q=0$.

What motivated the research which led to [1] was to reconcile these ideas with the globally well-defined supersymmetric F-theory solutions. This reconciliation has been successful and has lead to the conclusion that for $\operatorname{det} Q<0$ there are no well-defined 7-brane solutions while the branes with $\operatorname{det} Q>0$ play an important role in the construction of F-theory $(p, q)$ 7-brane solutions from a 10-dimensional point of view. In [1] it was further shown that the well-known F-theory 7-brane solutions form a subset of a much larger set of globally well-defined and supersymmetric 7-brane configurations. This larger set of solutions contains 7 -branes with $\operatorname{det} Q>0$. In this paper we study in more detail the properties of the 7-branes with $\operatorname{det} Q>0$. They will be referred to as 'Q7-branes'.

In the analysis of [1] a so-called pseudo-action (which includes 7-brane source terms) was used to describe the $\mathrm{SL}(2, \mathbb{R})$ invariant coupling of the 7-branes to the axion $\chi$ and the dilaton $\phi$ of IIB supergravity combined into a complex field $\tau=\chi+i e^{-\phi}$. One of the features of the pseudo-action of [1] is that the 7-brane source term does not contain a Wess-Zumino term describing the minimal electric coupling of the 7-brane to an 8-form field. The reason one can leave out such a term is because 8-forms are dual to the scalars and a source term for the scalars is provided by the Nambu-Goto term. If one then assumes holomorphicity of $\tau$ the Nambu-Goto source acts for both the real and imaginary part of $\tau$.

Part of the motivation for this article has been to improve on this situation by describing 7-brane coupling to an 8-form field via the Wess-Zumino term. We will explain how upon the duality transformation which eliminates the 8-form field, the information about

\footnotetext{
${ }^{1}$ It is understood that the $\operatorname{SL}(2, \mathbb{R})$ duality group is quantized and given by $\operatorname{SL}(2, \mathbb{Z})$. Whenever we speak of $\operatorname{SL}(2, \mathbb{R})$ we mean to imply that the result under discussion does not depend on the $\operatorname{SL}(2, \mathbb{Z})$ charge quantization.
} 
the magnetic coupling of the 7-brane to the axion-dilaton is encoded in a non-locality associated with the presence of a Dirac brane [5-8 stemmed from the 7-brane. The Dirac branes are associated with the branch cut properties of the holomorphic function $\tau$ describing the corresponding supersymmetric 7-brane solution.

Once we have identified the Q7-branes in the static limit with zero Born-Infeld (BI) vector fields we proceed to study the world-volume theory of the Q7-branes. It will be shown that the gauge invariant coupling of the Q7-brane to the IIB supergravity 8-, 6-, 4- and 2 -forms, described by the Wess-Zumino term, requires the introduction of two distinct BI fields. The two distinct BI vectors transform as a linear doublet under $\operatorname{SL}(2, \mathbb{R})$. We further argue that both of these BI fields are propagating on the world-volume by constructing the Dirac-Born-Infeld action up to second order in the BI vectors.

The paper is organized as follows. In section 2 we discuss in detail how a D7-brane couples electrically to the RR 8-form and magnetically to the $\mathrm{RR}$ axion via the presence of a Dirac 8-brane. This sets the stage for the discussion of the coupling of the Q7-branes in section 3. To describe the Q7-brane coupling new coordinates, denoted by $T$ and $\chi^{\prime}$, for the axion-dilaton coset manifold $\mathrm{SL}(2, \mathbb{R}) / \mathrm{SO}(2)$ are introduced. In section $\mathbb{t}$ we discuss the relation between the Dirac brane stemming from the Q7-brane and the monodromy of the axion-dilaton field $\tau$ measured when going around a Q7-brane. In section 5 the relation between the axion-dilaton fields $(\chi, \phi)$ and the fields $\left(\chi^{\prime}, T\right)$ is derived. In section 6 we describe how the Q7-branes couple to the 8-, 6-, 4- and 2-forms and to the axion-dilaton and in section 0 we end up with a summary of the results and with a discussion of some issues regarding the nature of the Q7-branes.

\section{D7-brane coupling}

To illustrate the method of how to couple magnetically charged branes to IIB supergravity it is instructive to first consider the example of the conventional Dirichlet 7-brane. In this particular case the coupling to the corresponding sector of IIB supergravity follows the classical rules of how to describe magnetically charged particles à la the Dirac monopole [5]. This has been extended to cases of various magnetically charged brane sources in [6-8].

The D7-brane world-volume action in the Einstein frame (which is appropriate for our purposes) has the following form [9]

$$
S=-\int_{\mathcal{M}_{8}} d^{8} \xi e^{\phi} \sqrt{-\operatorname{det}\left(g_{\mu \nu}+e^{-\frac{1}{2} \phi} \mathcal{F}_{\mu \nu}\right)}-\int_{\mathcal{M}_{8}} \mathcal{C} \wedge e^{\mathcal{F}_{2}},
$$

where $\mathcal{M}_{8}$ is the 8 -dimensional world-volume parameterized by $\xi^{\mu}$, with $\mu=0,1, \ldots, 7$ and where $\phi(\hat{x}(\xi))$ and $g_{\mu \nu}$ are the pullbacks of the dilaton and the target space metric onto the world-volume, respectively. In the Wess-Zumino term $\mathcal{C}$ denotes the formal sum of the pullbacks of the (duality related) RR potentials $C_{r}(\hat{x}(\xi))(r=0,2,4,6,8)$ and $\mathcal{F}_{2}=d \mathcal{A}_{1}+B_{2}$ is the field strength of the world-volume Born-Infeld gauge field $\mathcal{A}_{1}$ extended with the pullback of the NSNS 2-form $B_{2}$.

In what follows, we shall discuss the coupling of the brane action in which the BornInfeld field has been put to zero, i.e. $\mathcal{F}_{2}=0$. In other words the D7-brane will be coupled 
only to the RR 8-form potential $C_{8}$ dual to the axion field $C_{0}=: \chi$. The reason is that for the Q7-branes whose charges are not in the same $\mathrm{SL}(2, \mathbb{R})$ conjugacy class as those of the D7-brane, the complete Born-Infeld part of the action is unknown. We shall present the Q7-brane Wess-Zumino term with two Born-Infeld fields in section 6.

In the absence of the Born-Infeld field the action (2.1) reduces to

$$
S=-\int_{\mathcal{M}_{8}} d^{8} \xi e^{\phi} \sqrt{-g_{(8)}}-\int_{\mathcal{M}_{8}} C_{8}
$$

where $\sqrt{-g_{(8)}}$ is used to denote $\sqrt{-\operatorname{det} g_{\mu \nu}}$.

We would like to couple this action to a corresponding part of IIB supergravity action, assuming that the NSNS and RR 2-form fields are zero (which otherwise would produce sources for a world-volume gauge field).

Since in (2.2) instead of the axion we have its dual 8-form potential $C_{8}$, we are not allowed to take the IIB supergravity action with the axion-dilaton sector in the conventional form

$$
S=\int d^{10} x \sqrt{-g_{(10)}}\left(R-\frac{1}{2} \partial_{m} \phi \partial^{m} \phi-\frac{1}{2} e^{2 \phi} \partial_{m} \chi \partial^{m} \chi\right)
$$

where $C_{8}$ does not appear.

The issue is that we should work, both in the bulk as well as in the source term, with either $C_{8}$ or $\chi$. It seems natural to electrically couple the D7-brane to $C_{8}$, but most of the 7-brane calculations are done in a formulation in which the 7-brane magnetically couples to the axion. Therefore, the best strategy is not to work with either of the two formulations but instead take as the starting point the following first order action which, as we will see, interpolates between the two formulations:

$$
\begin{aligned}
S= & \int d^{10} x \sqrt{-g_{(10)}}\left(R-\frac{1}{2} \partial_{m} \phi \partial^{m} \phi-\frac{1}{2} e^{2 \phi} F_{m} F^{m}+\frac{1}{8 ! \sqrt{-g_{(10)}}} \epsilon^{m n_{1} \cdots n_{9}} F_{m} \partial_{n_{1}} C_{n_{2} \cdots n_{9}}\right) \\
& -\int_{\mathcal{M}_{8}} d^{8} \xi e^{\phi} \sqrt{-g_{(8)}}-\int_{\mathcal{M}_{8}} C_{8} .
\end{aligned}
$$

Here $F_{m}$ is now an auxiliary vector field which replaces the axion derivative and $C_{8}$ is its dual. Without the source term, eq. (2.4) establishes the standard duality relation between the fields $\chi$ and $C_{8}$.

To perform the duality transform in the presence of the brane source, we should rewrite the second line of eq. (2.4) as a 10D bulk integral. To this end we introduce the D7-brane current 8-form with the delta function having support on the D7-brane world-volume

$$
J^{m_{1} \cdots m_{8}}=\frac{1}{\sqrt{-g_{(10)}}} \int_{\mathcal{M}_{8}} d \hat{x}^{m_{1}} \wedge \cdots \wedge d \hat{x}^{m_{8}} \delta(x-\hat{x}(\xi)) .
$$

The 2-form dual of the current is the closed form

$$
\left({ }^{*} J\right)_{m_{1} m_{2}}=\frac{1}{8 !} \epsilon_{m_{1} m_{2} n_{1} \cdots n_{8}} \int_{\mathcal{M}_{8}} d \hat{x}^{n_{1}} \wedge \cdots \wedge d \hat{x}^{n_{8}} \delta(x-\hat{x}(\xi)), \quad d^{*} J_{8}=0 .
$$


As such, at least locally, we can represent ${ }^{*} J_{8}$ as the differential of a 1 -form which we shall call ${ }^{*} G_{9}$, namely

$$
{ }^{*} J_{8}=d{ }^{*} G_{9} \quad \Rightarrow \quad\left({ }^{*} G\right)_{m}=\frac{1}{9 !} \epsilon_{m n_{1} \cdots n_{9}} \int_{\mathcal{M}_{9}} d \hat{x}^{n_{1}} \wedge \cdots \wedge d \hat{x}^{n_{9}} \delta(x-\hat{x}(y))
$$

and

$$
G^{n_{1} \cdots n_{9}}=\frac{1}{\sqrt{-g_{10}}} \int_{\mathcal{M}_{9}} d \hat{x}^{n_{1}} \wedge \cdots \wedge d \hat{x}^{n_{9}} \delta(x-\hat{x}(y)) .
$$

In the last equation the delta function has the support on a 9-dimensional surface $\mathcal{M}_{9}$, parameterized by coordinates $y$, whose boundary is the world-volume $\mathcal{M}_{8}$ of the D7-brane. The 9-dimensional surface is associated with the world-volume of a Dirac 8-brane, which is a brane generalization of the Dirac string stemming from a monopole. In the present case we have a Dirac 8-brane stemming from the D7-brane. It is by means of the Dirac 8-brane that the D7-brane will magnetically couple to the axion field strength $F_{1}=d \chi$ as we shall see.

With the help of the dual current (2.6) the second line of eq. (2.4) can be rewritten as a 10D integral as follows

$$
\begin{aligned}
S= & \int_{\mathcal{M}_{10}} d^{10} x \sqrt{-g_{(10)}}\left(R-\frac{1}{2} \partial_{m} \phi \partial^{m} \phi-\frac{1}{2} e^{2 \phi} F_{m} F^{m}+\frac{1}{8 ! \sqrt{-g_{(10)}}} \epsilon^{m n_{1} \cdots n_{9}} F_{m} \partial_{n_{1}} C_{n_{2} \cdots n_{9}}\right) \\
& -\int_{\mathcal{M}_{10}} d^{10} x \int_{\mathcal{M}_{8}} d^{8} \xi \delta(x-\hat{x}(\xi)) e^{\phi} \sqrt{-g_{(8)}}-\int_{\mathcal{M}_{10}} C_{8} \wedge^{*} J_{8},
\end{aligned}
$$

where we use the convention that $\epsilon_{01 \ldots 9}=-\epsilon^{01 \ldots 9}=1$. We will use this first order action to derive expressions for the bulk plus source terms with $\chi$ or $C_{8}$ only.

We now wish to first eliminate from eq. (2.9) the field $C_{8}$ by solving the equations of motion of $F_{1}$ and $C_{8}$. The variation of (2.9) with respect to $F_{1}$ gives the duality condition

$$
F_{1}=e^{-2 \phi *} d C_{8}=: e^{-2 \phi *} F_{9} .
$$

The variation with respect to $C_{8}$ gives

$$
d F_{1}=-{ }^{*} J_{8} .
$$

Thus $F_{1}$ is not a closed form. However, recalling that the dual current $* J_{8}$ is the exact form (2.6), we have

$$
d F_{1}=-{ }^{*} J_{8}=-d^{*} G_{9} \quad \Rightarrow \quad d\left(F_{1}+{ }^{*} G_{9}\right)=0 .
$$

Hence, at least locally,

$$
F_{1}+{ }^{*} G_{9}=d \chi, \quad \Rightarrow \quad F_{1}=d \chi-{ }^{*} G_{9}
$$

where $\chi$ is the axion.

We can now eliminate the field $C_{8}$ from the action. To do this we note that up to a total derivative (which we shall skip) the last term in (2.9) can be rewritten as

$$
-\int_{\mathcal{M}_{10}} C_{8} \wedge{ }^{*} J_{8}=\int_{\mathcal{M}_{10}}\left(d C_{8} \wedge{ }^{*} G_{9}+\text { total derivative }\right) .
$$


Let us now substitute in the action (2.9) the expression (2.13) for the auxiliary field $F_{1}$. Then the term (2.14) and the last term in the first line of (2.9) cancel each other (up to a total derivative). As a result we arrive at the action

$$
S_{\mathrm{I}}=\int_{\mathcal{M}_{10}} d^{10} x \sqrt{-g_{(10)}}\left(R-\frac{1}{2} \partial_{m} \phi \partial^{m} \phi-\frac{1}{2} e^{2 \phi} F_{m} F^{m}\right)-\int_{\mathcal{M}_{8}} d^{8} \xi e^{\phi} \sqrt{-g_{(8)}},
$$

where $F_{1}=d x^{m} F_{m}$ is defined in (2.13). We see that the minimal coupling of the D7-brane to $C_{8}$ disappears and its role is taken upon by the non-local terms in the axion field strength $F_{1}$ due to the contribution of the Dirac 8-brane.

Alternatively, we can eliminate the axion in favor of the $\mathrm{RR}$ field $C_{8}$. To this end we apply the equation of motion corresponding to the auxiliary field $F_{m}$ leading to the duality relation (2.10). We use this relation to eliminate $F_{m}$ and this leads to the following action

$$
\begin{aligned}
S_{\mathrm{II}}= & \int d^{10} x \sqrt{-g_{(10)}}\left(R-\frac{1}{2} \partial_{m} \phi \partial^{m} \phi-\frac{1}{2 \cdot 9 !} e^{-2 \phi} F_{m_{1} \cdots m_{9}} F^{m_{1} \cdots m_{9}}\right) \\
& -\int_{\mathcal{M}_{8}} d^{8} \xi e^{\phi} \sqrt{-g_{(8)}}-\int_{\mathcal{M}_{8}} C_{8},
\end{aligned}
$$

where $F_{9}=d C_{8}$. Since the actions $S_{\mathrm{I}}$ and $S_{\mathrm{II}}$ follow from the same action $\mathrm{S}$ given in (2.9) they are classically equivalent.

\section{Q7-brane coupling}

In the generic case of a $(p, q, r)$ 7-brane (referred to as the Q7-brane) the Nambu-Goto and the Wess-Zumino part of the 7-brane action has the following form [12]

$$
S=-\int_{\mathcal{M}_{8}} d^{8} \xi T \sqrt{-g_{(8)}}-\int_{\mathcal{M}_{8}} q_{\alpha \beta} A_{8}^{\alpha \beta},
$$

where $T$ given by

$$
T=q_{\alpha \beta} V_{-}^{\alpha} V_{+}^{\beta}=\frac{1}{\operatorname{Im} \tau}\left(p+r \operatorname{Re} \tau+q|\tau|^{2}\right)
$$

is the so-called 'tension scalar' (for the definition of $V_{ \pm}^{\alpha}$ and related quantities we refer the reader to appendix $B$ ). In Subsection 5.1 it is shown that for $q \neq 0$ we have $\operatorname{sign}(q) T \geq$ $2 \sqrt{\operatorname{det} Q}$ so that the sign of the tension is determined by the sign of the parameter $q{ }^{2}$ The 8-forms $A_{8}^{\alpha \beta}$ form an SU(1,1) triplet among which only two 8-forms are independent due to the following constraint on their field strengths [3] (see also appendix G, eq. (C.16])

$$
V_{-}^{\alpha} V_{+}^{\beta} F_{9 \alpha \beta}=0 \text {. }
$$

The charge tensor $q_{\alpha \beta}$ transforms in the adjoint of $\mathrm{SU}(1,1)$. The $\mathrm{SU}(1,1)$ indices $\alpha, \beta$ are raised and lowered with the 2 -dimensional epsilon symbol $\epsilon_{12}=\epsilon^{12}=1$, i.e. $q^{\alpha}{ }_{\beta}=\epsilon^{\alpha \gamma} q_{\gamma \beta}$

\footnotetext{
${ }^{2}$ The tension $T$ of a 7 -brane is negative when $q<0$. The negative tension Q7-branes play a similar role as orientifold O8-planes play in the case of the D8-brane solutions [22]; they are used to make the supergravity solutions globally well-defined. Orientifold 7-planes only show up when the axion-dilaton field $\tau$ is constant 23. For the case of non-constant $\tau$ we need negative tension $Q 7$-branes [i].
} 
for raising and $q_{\alpha}{ }^{\beta}=q^{\gamma \beta} \epsilon_{\gamma \alpha}$ for lowering the indices. The action (3.1) is $\mathrm{SU}(1,1)$ invariant provided we also transform the charges $q_{\alpha \beta}$. We introduce the $\operatorname{SL}(2, \mathbb{R})$ algebra valued charge matrix $Q$ defined by

$$
Q=-\frac{i}{2} S^{-1} q \epsilon S=\left(\begin{array}{cc}
r / 2 & p \\
-q & -r / 2
\end{array}\right), \quad S=\left(\begin{array}{cc}
-i & 1 \\
i & 1
\end{array}\right),
$$

where $q \epsilon$ is a matrix whose components are given by $(q \epsilon)^{\alpha}{ }_{\beta}=q^{\alpha \gamma} \epsilon_{\gamma \beta}$. The matrix $Q$ was mentioned in the Introduction, eq. (1.1). The matrix $S$ establishes the isomorphism between $\mathrm{SL}(2, \mathbb{R})$ and $\mathrm{SU}(1,1)$.

Each value of $\operatorname{det} Q$ forms an $\operatorname{SL}(2, \mathbb{R})$ conjugacy class. The conjugacy classes of $\mathrm{SL}(2, \mathbb{R})$ are characterized by the value of the trace of the $\mathrm{SL}(2, \mathbb{R})$ matrix

$$
e^{Q}=\cos (\sqrt{\operatorname{det} Q}) \mathbb{1}+\frac{\sin (\sqrt{\operatorname{det} Q})}{\sqrt{\operatorname{det} Q}} Q .
$$

The families of conjugacy classes are formed by

$$
\operatorname{Tr} e^{Q}=2 \cos (\sqrt{\operatorname{det} Q})\left\{\begin{array} { l } 
{ = 2 } \\
{ > 2 } \\
{ < 2 }
\end{array} \text { or by } \operatorname { d e t } Q \left\{\begin{array}{l}
=0 \\
<0 \\
>0
\end{array}\right.\right.
$$

When $\operatorname{det} Q=0$ we are in the conjugacy class to which the $(p, q) 7$-branes belong. The D7-brane corresponds to $p=1$ and $q=r=0$.

\subsection{Q7-branes in the $\left(T, \chi^{\prime}\right)$ basis}

In the case of the D7-brane the RR 8-form is dual to the RR scalar and the RR scalar does not appear in the Nambu-Goto part, so that in some sense the degrees of freedom described by the Nambu-Goto and Wess-Zumino part are 'orthogonal'. In the case of the Q7-brane action (3.1) the Nambu-Goto term contains a non-linear combination $T=q_{\alpha \beta} V_{-}^{\alpha} V_{+}^{\beta}$ of the dilaton and axion fields. The question is whether we again have that the scalar which is dual to $q_{\alpha \beta} A_{8}^{\alpha \beta}$ and the scalar $T$ are independent or 'orthogonal'. Therefore one must know to which axion-dilaton function the field $q_{\alpha \beta} A_{8}^{\alpha \beta}$ is dual to and whether or not this composite scalar field appears as part of $T$ in the Nambu-Goto term. If the latter were the case, this would significantly complicate the dualization procedure, since it would then require the explicit use of the PST formalism [13] and the proof that the coupling of the 7-brane to the duality symmetric type IIB supergravity [14, 3] obeys the PST symmetries, as e.g. in the case of the M5-brane coupled to $11 \mathrm{D}$ supergravity [8].

As it turns out the tension scalar $T$ in the Nambu-Goto term is completely orthogonal (on the mass shell) to the scalar field which is the dual of $q_{\alpha \beta} A_{8}^{\alpha \beta}$, to be called $\chi^{\prime}$, in the sense that both have diagonal kinetic terms, see below. This diagonalization occurs due to the fact that the combination $V_{-}^{(\alpha} V_{+}^{\beta)}$, which appears in the definition of the scalar $T$, is orthogonal to $F_{9}^{\alpha \beta}$ by virtue of the constraint (3.3).

In order to rewrite the axion-dilaton kinetic terms in terms of $T$ and $q_{\alpha \beta} A_{8}^{\alpha \beta}$ we first observe that the derivative of $T$ can be written as

$$
d T=q_{\alpha \beta} V_{+}^{\alpha} V_{+}^{\beta} \bar{P}+q_{\alpha \beta} V_{-}^{\alpha} V_{-}^{\beta} P,
$$


where $P$ is defined in eq. (B.4). At the same time, the duality relation can be written as

$$
F_{9}^{\alpha \beta}=-i *\left(V_{-}^{\alpha} V_{-}^{\beta} P-V_{+}^{\alpha} V_{+}^{\beta} \bar{P}\right)
$$

see eq. (C.13). It is now straightforward to show that we can write the scalar field kinetic terms of the IIB supergravity action as

$$
\partial_{m} \phi \partial^{m} \phi+e^{2 \phi} \partial_{m} \chi \partial^{m} \chi=\frac{1}{T^{2}-4 \operatorname{det} Q}\left(\partial_{m} T \partial^{m} T+\frac{1}{9 !} q_{\alpha \beta} F_{m_{1} \cdots m_{9}}^{\alpha \beta} q_{\gamma \delta} F^{\gamma \delta m_{1} \cdots m_{9}}\right)
$$

where $F_{9}^{\alpha \beta}$ has been defined in (3.8). With this observation the dualization procedure of the coupled IIB supergravity - Q7-brane system proceeds analogously to the case of the D7-brane, but with the fields $T$ and $q_{\alpha \beta} A_{8}^{\alpha \beta}$ instead of $\phi$ and $C_{8}$. We start with the following first-order action which is similar to (2.9)

$$
\begin{aligned}
S= & \int_{\mathcal{M}_{10}} d^{10} x \sqrt{-g_{(10)}}\left(R-\frac{\partial_{m} T \partial^{m} T}{2\left(T^{2}-4 \operatorname{det} Q\right)}-\frac{1}{2}\left(T^{2}-4 \operatorname{det} Q\right) F_{m} F^{m}\right. \\
& \left.+\frac{1}{8 ! \sqrt{-g_{10}}} \epsilon^{m n_{1} \cdots n_{9}} F_{m} \partial_{n_{1}} q_{\alpha \beta} A_{n_{2} \cdots n_{9}}^{\alpha \beta}\right)-\int_{\mathcal{M}_{8}} d^{8} \xi T \sqrt{-g_{(8)}}-\int_{\mathcal{M}_{8}} q_{\alpha \beta} A_{(8)}^{\alpha \beta},
\end{aligned}
$$

with $F_{m}$ being an auxiliary vector field. We derive from this action the equations of motion for $F_{m}$ and $q_{\alpha \beta} A_{8}^{\alpha \beta}$ (which only appears in the first and the third term of the second line):

$$
d F_{1}=-{ }^{*} J_{8}=-d^{*} G_{9} \quad \Rightarrow \quad F_{1}=:\left(d \chi^{\prime}-{ }^{*} G_{9}\right),
$$

and

$$
{ }^{*} d\left(q_{\alpha \beta} A_{8}^{\alpha \beta}\right)={ }^{*} q_{\alpha \beta} F_{(9)}^{\alpha \beta}=\left(T^{2}-4 \operatorname{det} Q\right) F_{1} .
$$

These two equations implicitly define the axion $\chi^{\prime}$. Now substituting the solution (3.11) for $F_{1}$ back into the action (3.10) we get the analogue of (2.15)

$$
\begin{aligned}
S_{\mathrm{I}}= & \int_{\mathcal{M}_{10}} d^{10} x \sqrt{-g_{(10)}}\left(R-\frac{\partial_{m} T \partial^{m} T}{2\left(T^{2}-4 \operatorname{det} Q\right)}-\frac{1}{2}\left(T^{2}-4 \operatorname{det} Q\right) F_{m} F^{m}\right) \\
& -\int_{\mathcal{M}_{8}} d^{8} \xi T \sqrt{-g_{(8)}}
\end{aligned}
$$

where now $F_{1}=d x^{m} F_{m}=\left(d \chi^{\prime}-{ }^{*} G_{9}\right)$ and the Wess-Zumino term has disappeared.

On the other hand instead of using eq. (3.11) we can substitute in (3.10) the solution for $F_{1}$ in terms of the dual field strength ${ }^{*} q_{\alpha \beta} F_{9}^{\alpha \beta}$ (3.12) and obtain the local action describing the minimal coupling of the Q7-brane to the $\mathrm{SL}(2, \mathbb{R})$ invariant fields $T$ and $q_{\alpha \beta} A_{8}^{\alpha \beta}$

$$
\begin{aligned}
S_{\mathrm{II}}= & \int_{\mathcal{M}_{10}} d^{10} x \sqrt{-g_{(10)}}\left[R-\frac{1}{2\left(T^{2}-4 \operatorname{det} Q\right)}\left(\partial_{m} T \partial^{m} T\right.\right. \\
& \left.\left.+\frac{1}{9 !} q_{\alpha \beta} F_{m_{1} \cdots m_{9}}^{\alpha \beta} q_{\gamma \delta} F^{\gamma \delta m_{1} \cdots m_{9}}\right)\right]-\int_{\mathcal{M}_{8}} d^{8} \xi T \sqrt{-g_{(8)}}-\int_{\mathcal{M}_{8}} q_{\alpha \beta} A_{8}^{\alpha \beta} .
\end{aligned}
$$




\subsection{Unobservability of the Dirac 8-brane}

To describe the magnetic coupling of the 7-brane to the axion we have introduced into the actions (2.15) and (3.13) the Dirac 8-brane (2.7) and (2.8). As in the classical Dirac monopole problem, the Dirac brane is not a physical object, i.e. the dynamics of the system should not depend on the orientation of the Dirac 8-brane in the bulk. This is reflected in the fact that the 8-brane equations of motion are not independent. They are satisfied provided the axion field equations hold. To see this, let us derive the axion field equation and the equation of motion of the embedding coordinates of the Dirac 8-brane.

The field equation of $\chi^{\prime}$ is

$$
\partial_{m}\left[\sqrt{-g_{(10)}}\left(T^{2}-4 \operatorname{det} Q\right) F^{m}\right]=0 .
$$

It is accompanied by the Bianchi identity which follows from the definitions (3.11) and (2.7)

$$
d F_{1}=-{ }^{*} J_{8}
$$

with $J_{8}$ being the 7-brane current (2.5). Eqs. (3.15) and (3.16) describe the magnetic coupling of the 7-brane to $\chi^{\prime}$.

The variation of (3.13) with respect to the Dirac 8-brane world-volume coordinates $\hat{x}^{m}(y)$, appearing in (3.11) and (2.7), produces the equation

$$
\left.\partial_{m}\left(\sqrt{-g_{(10)}}\left(T^{2}-4 \operatorname{det} Q\right) F^{m}\right)\right|_{\mathcal{M}_{9}}=0,
$$

which is nothing but the field $\chi^{\prime}$ equation (3.15) pulled back on the Dirac 8-brane worldvolume. Therefore, the Dirac brane is not physical. Its position can be anywhere in space-time and it is invisible provided the Dirac veto holds, which does not allow the Dirac brane to intersect the world-volumes of the objects coupled to $\chi^{\prime}$ in an electric way. If such objects (which would be instantons) are present, their 'currents' contribute to the right hand side of eq. (3.15), while eq. (3.17) remains sourceless. The two equations are then consistent provided the world-volumes of the Dirac brane and the electrically charged objects never intersect. ${ }^{3}$ In quantum theory, as is well known, the unobservability of the Dirac branes is guaranteed by the Dirac quantization condition which results in the quantization of corresponding fluxes.

\subsection{Field equations and static 7-brane solutions}

Let us now consider the complete set of equations of motion which follow from the action (3.13). The variation with respect to the 10-dimensional metric results in the Einstein equations with the energy-momentum tensor $T_{m n}$ having the contributions from the axiondilaton and the 7-brane

$$
R_{m n}-\frac{1}{2} g_{m n} R=T_{m n}
$$

\footnotetext{
${ }^{3}$ Additional complications and subtleties regarding the Dirac branes and corresponding singular terms in the action and equations of motion arise when the action contains Wess-Zumino terms with 'bare' electric and/or magnetic potentials. In such cases it becomes much less trivial to reconcile the Dirac veto with the physical field equations. This happens for example in the case of the M5-brane [8]. In [16] a consistent method was developed to resolve these problems and related problems of anomalies.
} 
The variation with respect to the field $T$ gives

$\mathcal{D}_{m}\left(\frac{1}{T^{2}-4 \operatorname{det} Q} \partial^{m} T\right)+\frac{T \partial_{m} T \partial^{m} T}{\left(T^{2}-4 \operatorname{det} Q\right)^{2}}-T F_{m} F^{m}=\frac{1}{\sqrt{-g_{10}}} \int d^{8} \xi \sqrt{-g_{8}} \delta(x-\hat{x}(\xi))$.

The field equation of $\chi^{\prime}$ and the corresponding Bianchi identity have been given, respectively, in eqs. (3.15) and (3.16).

The 7-brane equation of motion obtained by varying (3.13) with respect to the worldvolume field $\hat{x}^{m}\left(\xi^{\mu}\right)$ is

$$
\begin{aligned}
& \operatorname{TD}_{\mu}\left(\sqrt{-g_{8}} g_{8}^{\mu \nu} \partial_{\mu} \hat{x}^{n}\right) g_{n m}^{10}-\sqrt{-g_{8}} \partial_{\mu} T \\
& \quad=\frac{1}{8 !}\left(T^{2}-4 \operatorname{det} Q\right) \epsilon^{\mu_{1} \cdots \mu_{8}} \partial_{\mu_{1}} x^{n_{1}} \cdots \partial_{\mu_{8}} x^{n_{8}} \epsilon_{n_{1} \cdots n_{8} m l} F^{l}(\hat{x}(\xi)),
\end{aligned}
$$

where $\mu, \nu=0,1, \cdots, 8$ are 7 -brane world-volume indices and $\mathcal{D}_{\mu}$ is the pullback of the 10D bulk covariant derivative containing the Christoffel symbols.

Let us now consider supersymmetric solutions of eqs. (3.18) to (3.20) corresponding to the 7-branes. The simplest possible assumption is that the 7-brane is static and does not fluctuate in the transverse directions $x^{8,9}=$ cst. We can also assume that the axion and dilaton fields depend only on the transverse coordinates $x^{i}(i=8,9)$. Such an Ansatz corresponds to the dimensional reduction of the supergravity - 7-brane system to a 2 dimensional system with the 7-brane being 'shrunk' to a point in the 2-dimensional space. The Dirac 8-brane then reduces to a Dirac string which ends on the pointlike particle counterpart of the 7-brane.

The consistency condition, which must hold in order that the 7-brane is static, is obtained by using the static gauge and setting in (3.20) the derivatives of the transverse scalars $\hat{x}^{8}$ and $\hat{x}^{9}$ equal to zero. It has the form

$$
\partial_{i} T=-\left(T^{2}-4 \operatorname{det} \mathrm{Q}\right) \sqrt{-\mathrm{g}} \epsilon_{01 \ldots 7 \mathrm{ij}}\left(\partial^{\mathrm{j}} \chi^{\prime}-\left(* \mathrm{G}_{(9)}\right)^{\mathrm{j}}\right),
$$

where $i, j=8,9$. Outside the source using that the metric longitudinal to the 7 -brane is flat (which follows from supersymmetry) we find

$$
\partial_{8} T=-\left(T^{2}-4 \operatorname{det} \mathrm{Q}\right) \partial_{9} \chi^{\prime}, \quad \partial_{9} \mathrm{~T}=\left(\mathrm{T}^{2}-4 \operatorname{det} \mathrm{Q}\right) \partial_{8} \chi^{\prime} .
$$

These equations can be used to construct the solution for $T$ and $\chi^{\prime}$ in the neighborhood of a 7-brane, i.e. near $z=0$. Since the axion describes the magnetic charge of the 7-brane, i.e. the axion charge equals

$$
m=\int_{0}^{2 \pi} \frac{d \chi^{\prime}}{d \theta} d \theta
$$

with $z=x^{8}+i x^{9}=r e^{i \theta}$, near the 7-brane we can take

$$
\chi^{\prime}=\frac{m}{2 \pi} \theta .
$$

Writing equations (3.22) in the form of the Cauchy-Riemann differential equations we find that for $\operatorname{det} Q=0$ near $z=0$

$$
\tau=\chi^{\prime}+\frac{i}{T}=\frac{m}{2 \pi i} \log z
$$


while for $\operatorname{det} Q>0$ near $z=0$ we have

$$
\mathcal{T}=\chi^{\prime}+\frac{i}{4 \sqrt{\operatorname{det} Q}} \log \frac{T+2 \sqrt{\operatorname{det} Q}}{T-2 \sqrt{\operatorname{det} Q}}=\frac{m}{2 \pi i} \log z .
$$

In both cases, solutions (3.25) and (3.26), the axion charge $m$ is equal to the number of 7-branes which are located at the point $z=0$.

In footnote 2 it is mentioned that for $q<0$ the tension of a 7 -brane can be negative and that negative tension Q7-branes are used in the construction of globally well-defined 7-brane solutions. So we would like to include them as potential source terms to the IIB supergravity action. It can be seen from eq. (3.26) that near $z=0$ the tension $T$ of a Q7-brane will be negative whenever $m<0$. We will however prefer to keep $m>0$ so that for a positive/negative tension Q7-brane we have

$$
\operatorname{sign}(q) \mathcal{T}=\frac{m}{2 \pi i} \log z .
$$

The condition (3.21) under which the 7-brane can be considered static coincides with the condition that $\tau$ or $\mathcal{T}$ are holomorphic functions with logarithmic branch cuts. As it follows from equation (3.21), along the Dirac string the holomorphicity fails, so the Dirac string plays the role of a branch cut of $\tau$ or $\mathcal{T}$. Crossing of these branch cuts is related to the nontrivial monodromy of the functions $\tau$ and $\mathcal{T}$. This will be the subject of the next section.

\section{Dirac strings and monodromy}

\subsection{Q7-branes in the $(\tau, \bar{\tau})$ basis}

Let us now consider how a Q7-brane, magnetically coupled to the field $\chi^{\prime}$, couples to the conventional axion and dilaton $(\chi, \phi)$ or rather to $(\tau, \bar{\tau})$. The duality relations (3.11), (3.12) and (3.8) (taken in the absence of the Dirac brane) prompt us that the differential of $\chi^{\prime}$ is expressed in terms of the $\mathrm{SU}(1,1) / \mathrm{U}(1)$ Cartan forms (B.4) and (B.5) as follows

$$
\left(T^{2}-4 \operatorname{det} Q\right) d \chi^{\prime}=-i q_{\alpha \beta}\left(V_{-}^{\alpha} V_{-}^{\beta} P-V_{+}^{\alpha} V_{+}^{\beta} \bar{P}\right)=q_{\alpha \beta}{ }^{*} F_{9}^{\alpha \beta} .
$$

We substitute this expression for $d \chi^{\prime}$ into the action (3.13) and subsequently use eq. (3.2) and eqs. (B.8) to (B.10) of appendix B. This yields the following action for the coupling of the Q7-brane to the standard form of the IIB supergravity action

$$
\begin{aligned}
S= & \int_{\mathcal{M}_{10}} d^{10} x \sqrt{-g_{(10)}}\left[R-\frac{1}{2(\operatorname{Im} \tau)^{2}}\left|\partial_{m} \tau+\left(p+q \tau^{2}+r \tau\right)\left(* G_{9}\right)_{m}\right|^{2}\right] \\
& -\int_{\mathcal{M}_{8}} d^{8} \xi \frac{1}{\operatorname{Im} \tau}\left(p+q|\tau|^{2}+r \frac{\tau+\bar{\tau}}{2}\right) \sqrt{-g_{(8)}} .
\end{aligned}
$$

This action also applies when $\operatorname{det} Q=0$. For the case $p=1$ and $q=r=0$ it can be seen to coincide with (2.15). 
Another way to write the action (4.2) is as follows

$$
S=\int_{\mathcal{M}_{10}} d^{10} x \sqrt{-g_{(10)}}\left(R-2 \hat{P}_{m}^{*} \hat{P}^{m}\right)-\int_{\mathcal{M}_{10}} d^{10} x \int_{\mathcal{M}_{8}} d^{8} \xi \delta(x-\hat{x}(\xi)) q_{\alpha \beta} V_{-}^{\alpha} V_{+}^{\beta} \sqrt{-g_{(8)}},
$$

where $\hat{P}_{m}$ is

$$
\hat{P}_{m}=P_{m}-\frac{i}{2} q_{\alpha \beta} V_{+}^{\alpha} V_{+}^{\beta}\left(* G_{9}\right)_{m} .
$$

In terms of $\hat{P}$ the duality relation between the 8 -form and $\chi^{\prime}$ field strengths in the presence of sources takes the form

$$
q_{\alpha \beta} F_{9}^{\alpha \beta}=-i *\left(q_{\alpha \beta} V_{-}^{\alpha} V_{-}^{\beta} \hat{P}-q_{\alpha \beta} V_{+}^{\alpha} V_{+}^{\beta} \hat{P}^{*}\right) .
$$

This relates the equations of motion and the Bianchi identity of the 8-form $q_{\alpha \beta} A_{8}^{\alpha \beta}$ to the Bianchi identity and the equations of motion of the axion $\chi^{\prime}$.

The Bianchi identity for $\hat{P}_{m}$ can be written as

$$
\hat{D} \hat{P}=: d \hat{P}-2 i \hat{\mathcal{Q}} \wedge \hat{P}=-\frac{i}{2} q_{\alpha \beta} V_{+}^{\alpha} V_{+}^{\beta} * J_{8},
$$

where

$$
\hat{\mathcal{Q}}_{m}=\mathcal{Q}_{m}+\frac{1}{2} q_{\alpha \beta} V_{+}^{\alpha} V_{-}^{\beta}\left(* G_{9}\right)_{m}
$$

and $\mathcal{Q}_{m}$ is a composite $\mathrm{U}(1)$ gauge field defined in (B.6). eq. (4.6) is the 'sourced' version of the Bianchi identity (B.7), i.e. of $D P=d P-2 i \mathcal{Q} \wedge P=0$.

\subsection{Monodromy}

The hatted 1-form fields $\hat{P}$ and $\hat{\mathcal{Q}}$ defined in (4.4) and (4.7) can be collected into the matrix-valued 1-form

$$
V\left(\begin{array}{cc}
-i \hat{\mathcal{Q}} & \hat{P} \\
\hat{\hat{P}} & i \hat{\mathcal{Q}}
\end{array}\right) V^{-1}=d V V^{-1}+S Q S^{-1} * G_{9},
$$

with $S$ defined in eq. (3.4) and where $V$ is the matrix defined in equation (B.2). Let us define

$$
\hat{p}=p+S Q S^{-1} * G_{9},
$$

where

$$
p=V\left(\begin{array}{cc}
0 & P \\
\bar{P} & 0
\end{array}\right) V^{-1} .
$$

Then $\hat{p}$ satisfies the Bianchi identity

$$
d \hat{p}-2 p \wedge p=S Q S^{-1} * J_{8} .
$$

Outside the source the Bianchi identity (4.11) is solved by

$$
p=\frac{1}{2}(d C) C^{-1},
$$


where $C=V V^{\dagger} .{ }^{4}$ Alternatively, we can write this solution as

$$
D C=0 \quad \text { with } \quad D=d-2 p .
$$

This equation can be interpreted as saying that $C$ is parallel transported with respect to the flat connection $p$. Let $\gamma(\lambda)$ be some path parameterized by $\lambda$ which runs from 0 to 1 . Then we have

$$
C(\lambda=1)=\mathcal{P} \exp \left[2 \int_{\gamma} p\right] C(\lambda=0)
$$

where $\mathcal{P}$ denotes the path ordering symbol. Since the connection is flat the quantity $\mathcal{P} \exp \left[2 \int_{\gamma} p\right]$ for closed $\gamma$ will only depend on the base point of the closed path. The location of the base point can be changed by a similarity transformation,

$$
\mathcal{P} \exp \left[2 \oint_{\gamma} p\right] \rightarrow H \mathcal{P} \exp \left[2 \oint_{\gamma} p\right] H^{-1} \quad \text { where } H=\mathcal{P} \exp \left[2 \int_{\tilde{\gamma}} p\right]
$$

with the path $\tilde{\gamma}$ connecting the initial to the final base point. This means that the eigenvalues of the monodromy matrix, $\mathcal{P} \exp \left[2 \oint_{\gamma} p\right]$, are preserved under shifting the position of the base point. Therefore a physical quantity that we can associate with the Bianchi identity $d p-2 p \wedge p=0$ is the Wilson line ${ }^{5}$

$$
\operatorname{Tr} \mathcal{P} \exp \left[2 \oint_{\gamma} p\right] .
$$

For 7-brane solutions the matrix $p$ only depends on the two coordinates transverse to the brane. In that case the quantity $\mathcal{P} \exp \left[2 \oint_{\gamma} p\right]$ will determine the monodromy of $C$ and thus of the scalars which parameterize it.

The monodromy of the matrix $C=V V^{\dagger}$ is given by

$$
C(\lambda=1)=\mathcal{P} \exp \left[2 \oint_{\gamma} p\right] C(\lambda=0) .
$$

Let us consider a path $\gamma$ which encircles the 7-brane (point) source in the transverse space. Further we assume that $\gamma$ encloses an area of infinitesimal size, denoted by $D$. Expanding the path-ordered expression up to second order we find

$$
C(\lambda=1)=\left(1+\int_{D}(d \hat{p}-2 p \wedge p)+\ldots\right) C(\lambda=0)\left(1+\int_{D}(d \hat{p}-2 p \wedge p)^{\dagger}\right),
$$

where we have used the fact that $p C=C p^{\dagger}$ and $\oint_{\gamma} p=\int_{D} d \hat{p}$. According to eq. (4.11) this can be written as

$$
C(\lambda=1)=\left(1+S Q S^{-1}+\ldots\right) C(\lambda=0)\left(1+\left(S Q S^{-1}\right)^{\dagger}+\ldots\right) .
$$

\footnotetext{
${ }^{4}$ The relation between the matrix $C$ and the matrix $M=e^{\phi}\left(\begin{array}{cc}|\tau|^{2} & \chi \\ \chi & 1\end{array}\right)$ is given by $M=S^{-1} C S$ with $S$ given in (3.4).

${ }^{5}$ The terminology is borrowed from Yang-Mills theory. Here $p$ is not a gauge field. It is because of a mathematical similarity that we call this quantity a Wilson line.
} 
Since we know the monodromy of $C=V V^{\dagger}$ when going at an infinitesimal distance around a 7 -brane and since we know the parametrization of $V$ in terms of $\tau$, see eq. (B.8), we know the monodromy of $\tau$. It follows that $\tau$ transforms as

$$
\tau \rightarrow e^{Q} \tau \text {. }
$$

The Wilson line (4.16) when evaluated around the contour $\gamma$ encircling a 7-brane at an infinitesimal distance can be evaluated and is equal to $\operatorname{Tr} e^{Q}$. Hence, the Wilson line computes what is called the $\mathrm{SL}(2, \mathbb{R})$ conjugacy class (see Subsection 5.1 for more details about the $\mathrm{SL}(2, \mathbb{R})$ conjugacy classes).

It was mentioned that in general the monodromy of the matrix $C$ and thus of $\tau$ is base point dependent. When $\tau$ is an analytic function (as in the case of 7-brane solutions) with a given monodromy around some closed contour $\gamma$, it must have a branch cut. In this case the base point dependence relates to the ordering of these branch cuts.

\subsection{Multiple 7-branes}

The action describing the coupling to the IIB supergravity axion-dilaton sector of $n 7$ branes is given by

$$
\begin{aligned}
S= & \int_{\mathcal{M}_{10}} d^{10} x \sqrt{-g_{(10)}}\left(R-2 \hat{P}_{m}^{*} \hat{P}^{m}\right) \\
& -\int_{\mathcal{M}_{10}} d^{10} x \sum_{k=1}^{n} \int_{\mathcal{M}_{8}^{k}} d^{8} \xi_{k} \delta\left(x-\hat{x}_{k}\left(\xi_{k}\right)\right) q_{\alpha \beta}^{k} V_{-}^{\alpha} V_{+}^{\beta} \sqrt{-g_{(8)}^{k}},
\end{aligned}
$$

with

$$
\hat{P}_{m}=P_{m}-\frac{i}{2} \sum_{k=1}^{n} q_{\alpha \beta}^{k} V_{+}^{\alpha} V_{+}^{\beta}\left(* G_{9}^{k}\right)_{m} .
$$

The world-volume $\mathcal{M}_{8}^{k}$ of each 7-brane, carrying a charge $q_{\alpha \beta}^{k}$, is parameterized by $\xi_{k}^{\mu}$ and is located in target space at the point $\hat{x}_{k}\left(\xi_{k}\right)$. Its embedding metric is $g_{\mu \nu}^{k}$ and the Dirac 8-brane stemming from the 7-brane is described by $G_{9}^{k}$.

When Dirac strings stemming from 7-branes with different charge matrices $Q$ intersect there will generically be a nontrivial monodromy for $\tau$ when going around the intersection point. The intersection point of two Dirac strings however does not describe the locus of another 7-brane, so we demand that Dirac strings can only intersect when the total monodromy measured when going around the intersection point is the identity.

Consider figure 11 which shows two intersecting Dirac strings stemming from different 7-branes characterized by the charge matrices $Q_{1}$ and $Q_{2}$. The point $b$ is taken as the base point to evaluate the monodromy of $\tau$ when going around the intersection point. The measured monodromy around the intersection point is

$$
e^{Q_{2}} e^{Q_{1}} e^{-Q_{2}} e^{-Q_{1}} .
$$

Since there is no brane located at the intersection point by assumption we must have that the monodromy (4.23) is equal to identity. This is only possible when

$$
\left[Q_{1}, Q_{2}\right]=0 .
$$




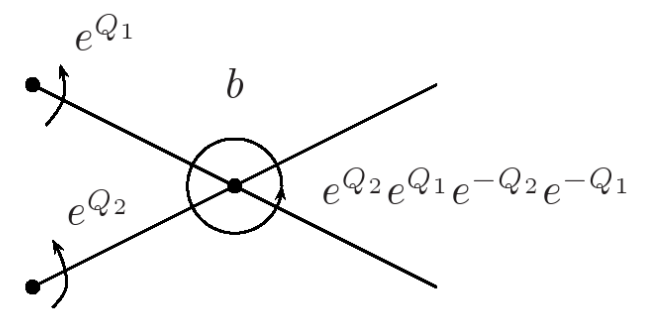

Figure 1: Two intersecting Dirac strings stemming from 7-branes with charge matrices $Q_{1}$ and $Q_{2}$. The point $b$ is taken as the base point for the monodromy of $\tau$ around the intersection point.

It can be seen that the condition (4.24) is base point independent. Hence, the Dirac strings of two 7-branes whose charge matrices are not proportional to each other $\left(Q_{1} \neq \alpha Q_{2}\right)$ are not allowed to intersect.

The fact that the Dirac strings of two 7-branes for which $Q_{1} \neq \alpha Q_{2}$ are not allowed to intersect each other is potentially worrisome because the Dirac strings are defined on the 2-dimensional transverse space and hence always intersect each other, if not at some finite point then at infinity. However, it is possible for three different Dirac strings to intersect each other as we will discuss next.

Consider the case of three different 7-branes with charges $Q_{1}, Q_{2}$ and $Q_{3}$ such that

$$
\left[Q_{1}, Q_{2}\right] \neq 0, \quad\left[Q_{1}, Q_{3}\right] \neq 0, \quad\left[Q_{2}, Q_{3}\right] \neq 0,
$$

but which satisfy

$$
e^{Q_{1}} e^{Q_{2}} e^{Q_{3}}=\mathbb{1}
$$

Then it is allowed for the collection of all three Dirac strings to intersect each other at one point. This property of three different Dirac strings is the basis of the construction of the globally well-defined 7-brane solutions of [1] where it is shown that any 7-brane configuration can be obtained by taking combinations of, what are referred to as, the $1 \mathrm{~A}$ and 1B buidling blocks. Each of these building blocks consists of three 7-branes.

\section{Conjugacy class dependence of IIB supergravity}

In section 3.1 the scalars $T$ and $\chi^{\prime}$ were introduced. In this section we will discuss in detail the geometrical nature of the relation between the two sets of the scalar fields $(\tau, \bar{\tau})$ and $\left(T, \chi^{\prime}\right)$. The IIB action in terms of $T$ and $\chi^{\prime}$, eq. (3.13) (without source terms), contains the parameter $\operatorname{det} Q$, which labels the $\operatorname{SL}(2, \mathbb{R})$ conjugacy classes. As long as the action is written in terms of scalars and not in terms of 8-forms the parameter $\operatorname{det} Q$ can be transformed away by an appropriate field redefinition. When the axion $\chi^{\prime}$ has been dualized into the 8form $q_{\alpha \beta} A_{8}^{\alpha \beta}$ it is no longer possible to transform away the conjugacy class label $\operatorname{det} Q$, i.e. we cannot by means of a local field redefinition go from the action (3.14) (without source terms) to the action (2.16) (without source terms). Whenever the conjugacy class label $\operatorname{det} Q$ cannot be transformed away by (local) field redefinitions we say that the resulting 
system is manifestly conjugacy class dependent. Of course another obvious situation in which one cannot transform away the parameter $\operatorname{det} Q$ is when one couples a Q7-brane to the IIB action.

\subsection{Coordinate systems on the scalar manifold}

From eq. (3.13) it follows that in the absence of the 7-brane sources the kinetic term for the scalar fields $\chi^{\prime}$ and $T$ has the form

$$
\mathcal{L}_{\text {scalar KT }}=-\sqrt{-g}\left(\frac{1}{2} \frac{1}{T^{2}-4 \operatorname{det} Q} \partial_{m} T \partial^{m} T+\frac{1}{2}\left(T^{2}-4 \operatorname{det} Q\right) \partial_{m} \chi^{\prime} \partial^{m} \chi^{\prime}\right) .
$$

The scalar field kinetic terms can be read as a line element of the space $\operatorname{SL}(2, \mathbb{R}) / \operatorname{SO}(2)$. This is a maximally symmetric space which has three Killing vectors. The Killing vectors are differential operators which generate the Lie algebra of $\operatorname{SL}(2, \mathbb{R})$. Along an integral curve generated by a Killing vector the metric does not change. In an adapted coordinate system one of the coordinates, here $\chi^{\prime}$, of the line element runs along such an integral curve. The presence of the Killing symmetry associated with the shift of $\chi^{\prime}$ is expressed by the fact that the metric components do not depend on $\chi^{\prime}$. We thus see that the shift symmetry of $\chi^{\prime}$ in adapted coordinates corresponds to an $\operatorname{SL}(2, \mathbb{R})$ transformation $\tau \rightarrow e^{Q} \tau$ of the axion-dilaton field.

In eq. $(3.26)$ we introduced a new complex field $\mathcal{T}$. In terms of this complex field the scalar field kinetic part of the IIB supergravity action (5.1) takes the form

$$
\mathcal{L}_{\mathcal{T}}=-\sqrt{-g} \frac{1}{2} \frac{\operatorname{det} Q \partial_{m} \mathcal{T} \partial^{m} \overline{\mathcal{T}}}{4 \sinh ^{2}(2 \sqrt{\operatorname{det} Q} \operatorname{Im} \mathcal{T})}
$$

while in terms of $\tau$ it is given by

$$
\mathcal{L}_{\tau}=-\sqrt{-g} \frac{1}{2} \frac{\partial_{m} \tau \partial^{m} \bar{\tau}}{(\operatorname{Im} \tau)^{2}}
$$

Comparing (5.2) and (5.3) one finds the following relation between $\tau$ and $\mathcal{T}^{6}$

$$
w=: \frac{\tau-\tau_{0}}{\tau-\bar{\tau}_{0}}=e^{2 i \operatorname{sign}(q) \sqrt{\operatorname{det} \mathrm{Q}} \mathcal{T}},
$$

where $\tau_{0}$ is given by

$$
\tau_{0}=-\frac{r}{2 q}+\frac{i}{|q|} \sqrt{\operatorname{det} \mathrm{Q}} .
$$

The point $\tau_{0}$ is a fixed point under the $e^{Q}$ transformation, i.e. $e^{Q} \tau_{0}=\tau_{0}$ for $\operatorname{det} Q \geq 0 .^{7}$ eq. (5.4) also defines the complex scalar $w$. From eqs. (5.4) and (3.26) we find that $T$ and

\footnotetext{
${ }^{6}$ The relation between $\tau$ and $\mathcal{T}$ could also have been obtained from the local solutions for $\tau$ and $\mathcal{T}$ near a Q7-brane. From eq. (3.27) we know that $z=e^{2 \pi i \operatorname{sign}(q) \mathcal{T} / m}$ while from 11 we know that near a Q7-brane we have $z=\left(\frac{\tau-\tau_{0}}{\tau-\bar{\tau}_{0}}\right)^{\frac{\pi}{\sqrt{\operatorname{det} Q}}}$ so that for $m=1$ (one Q7-brane) we obtain the relation (5.4).

${ }^{7}$ The action of a matrix $\Lambda=\left(\begin{array}{ll}a & b \\ c & d\end{array}\right)$ on $\tau$, written as $\Lambda \tau$, is defined as $\Lambda \tau=\frac{a \tau+b}{c \tau+d}$.
} 
$\chi^{\prime}$ are given by the following expressions in terms of $\tau$ and $\tau_{0}$

$$
\begin{aligned}
T & =\frac{1}{\operatorname{Im} \tau}\left(q|\tau|^{2}+r \operatorname{Re} \tau+p\right), \\
e^{4 i \operatorname{sign}(q) \sqrt{\operatorname{det} Q} \chi^{\prime}} & =\frac{|\tau|^{2}-2 \tau_{0} \operatorname{Re} \tau+\tau_{0}^{2}}{|\tau|^{2}-2 \bar{\tau}_{0} \operatorname{Re} \tau+\bar{\tau}_{0}^{2}} .
\end{aligned}
$$

The sign of the tension $T$ equals the sign of the parameter $q$ as follows from the following way of writing $T$ in terms of $(\tau, \bar{\tau})$

$$
T=\frac{q}{2 \operatorname{Im} \tau}\left[\left|\tau-\tau_{0}\right|^{2}+\left|\tau-\bar{\tau}_{0}\right|^{2}\right] .
$$

The transformation from $\tau$ to $\mathcal{T}$ is a conformal mapping. Consider the sequence

$$
\tau:(\text { upper half }- \text { plane }) \longrightarrow w:(\text { unit disk }) \longrightarrow \mathcal{T}:(\text { vertical strip })
$$

We first map $\tau$, which takes value in the upper half plane $(\operatorname{Im} \tau>0)$, to $w$ which parameterizes the unit disk $(|w|<1)$. Then we map this to $e^{2 i \operatorname{sign}(q) \sqrt{\operatorname{det} Q} \mathcal{T}}$ a vertical strip in a new upper half plane $(\operatorname{sign}(q) \operatorname{Im} \mathcal{T}>0)$. The region $\operatorname{Im} \mathcal{T}>0$ corresponds to $2 \sqrt{\operatorname{det} Q}<\operatorname{sign}(q) T<\infty$. The real line $\operatorname{Im} \tau=0$ gets mapped to the unit circle $|w|=1$ and subsequently to the real $\operatorname{line} \operatorname{Im} \mathcal{T}=0$ which however in the process has become periodically identified, $\chi^{\prime} \sim \chi^{\prime}+\frac{\pi}{\sqrt{\operatorname{det} Q}}$. Hence, the scalar field redefinition can be read as a conformal mapping from the upper half-plane $\operatorname{Im} \tau>0$ to the vertical strip $\operatorname{sign}(q) \operatorname{Im} \mathcal{T}>0$ with $\chi^{\prime} \sim \chi^{\prime}+\frac{\pi}{\sqrt{\operatorname{det} Q}}$.

In the $(\mathcal{T}, \overline{\mathcal{T}})$ coordinates the $\operatorname{SL}(2, \mathbb{R})$ symmetry is no longer manifest. The global symmetries are

$$
e^{2 i \operatorname{sign}(q) \sqrt{\operatorname{det} \mathrm{Q}} \mathcal{T}} \rightarrow \frac{\alpha e^{2 i \operatorname{sign}(q) \sqrt{\operatorname{det} \mathrm{Q}} \mathcal{T}}+\beta}{\bar{\beta} e^{2 i \operatorname{sign}(q) \sqrt{\operatorname{det} \mathrm{Q}} \mathcal{T}}+\bar{\alpha}} \quad \text { with } \quad|\alpha|^{2}-|\beta|^{2}=1 .
$$

It is not possible to realize the global symmetry directly on $\mathcal{T}$. The only manifest global symmetry left is the axion $\chi^{\prime}$ shift symmetry. We have for $\operatorname{det} Q>0$ that $w$ transforms as a U(1) 'matter' field

$$
w \rightarrow e^{2 i \operatorname{sign}(q) \sqrt{\operatorname{det} Q}} w \quad \text { when } \quad \tau \rightarrow e^{Q} \tau .
$$

It then follows from Eq. (5.4) that an arbitrary $\operatorname{det} Q>0$ transformation $\tau \rightarrow e^{Q} \tau$ can be written as $\chi^{\prime} \rightarrow \chi^{\prime}+\operatorname{sign}(q)$.

In section 6 we will construct the Q7-brane Wess-Zumino and for this purpose it is convenient to introduce a complex linear combination of the RR and NSNS 2-form fields $C_{2}$ and $B_{2}$ which transforms as (5.11) under the action of $e^{Q}$. We define the following complex 2-form $\mathcal{A}_{2}$

$$
\mathcal{A}_{2}=: \frac{-i}{\left(\operatorname{Im} \tau_{0}\right)^{1 / 2}}\left(-C_{2}+\tau_{0} B_{2}\right) \text {. }
$$

Using that the 2-forms transform under $e^{Q}$ as (see (C.28)

$$
\left(\begin{array}{l}
C_{2} \\
B_{2}
\end{array}\right) \rightarrow e^{Q}\left(\begin{array}{l}
C_{2} \\
B_{2}
\end{array}\right)
$$


we see that the field $\mathcal{A}_{2}$ transforms under $e^{Q}$ with $\operatorname{det} Q>0$ as

$$
\mathcal{A}_{2} \rightarrow e^{i \operatorname{sign}(q) \sqrt{\operatorname{det} Q}} \mathcal{A}_{2}
$$

With the use of eqs. (5.4) to (5.7) the duality relation (4.1) between the 8-form and the axionic scalar $\chi^{\prime}$ takes the form

$$
\left(T^{2}-4 \operatorname{det} Q\right) d \chi^{\prime}=\frac{\operatorname{det} Q}{\left(\operatorname{Im} \tau_{0}\right)^{2}}\left|\left(\tau-\tau_{0}\right)\left(\tau-\bar{\tau}_{0}\right)\right|^{2} d \chi^{\prime}=* q_{\alpha \beta} F_{9}^{\alpha \beta},
$$

where

$$
d \chi^{\prime}=\frac{\operatorname{Im} \tau_{0}}{2 \sqrt{\operatorname{det} Q}}\left[\frac{d \tau}{\left(\tau-\tau_{0}\right)\left(\tau-\bar{\tau}_{0}\right)}+\text { c.c. }\right]
$$

can be obtained by differentiating eq. (5.4). In appendix Q, eq. (C.18), we present the entire bosonic part of IIB supergravity action in which both $q_{\alpha \beta} F_{9}^{\alpha \beta}$ and $\chi^{\prime}$ appear. Since in the action (C.18) the parameter $\operatorname{det} Q$ appears explicitly (it also appears in $G_{7}$ ) and because it cannot be transformed away by a local field redefinition as discussed in the introduction to this section this way of writing the IIB supergravity action is referred to as a conjugacy class dependent formulation.

The $\operatorname{det} Q \rightarrow \mathbf{0}$ limit. One can take at any stage the limit $\operatorname{det} Q \rightarrow 0$. Since at various places, e.g. in the definition of $\tau_{0}$, eq. (5.5), we divide by $q$ one must assume that $q \neq 0$. This means that when one takes the limit $\operatorname{det} Q=p q-\frac{r^{2}}{4} \rightarrow 0$ it must be assumed that $q \neq 0$. Hence after taking this limit one ends up with a $(p, q)$ 7-brane for which $q \neq 0$. In order to get to the D7-brane one must perform an $\operatorname{SL}(2, \mathbb{R})$ transformation which takes one from a $(p, q) 7$-brane to a $(1,0) 7$-brane. The $\operatorname{det} Q \rightarrow 0$ limit of $\left(T, \chi^{\prime}\right)$ leads to the expressions

$$
\begin{aligned}
T & =\frac{1}{\operatorname{Im} \tau}\left(q|\tau|^{2}+r \operatorname{Re} \tau+p\right), \\
\chi^{\prime} & =-\frac{\frac{r}{2 q}+\operatorname{Re} \tau}{q|\tau|^{2}+r \operatorname{Re} \tau+p}, \quad p q=\frac{r^{2}}{4} .
\end{aligned}
$$

These two equations can be combined into the complex equation

$$
\mathcal{T}=\chi^{\prime}+i T^{-1}=\frac{-1}{q \tau+\frac{r}{2}}
$$

Hence, for $\operatorname{det} Q=p q-\frac{r^{2}}{4}=0$ the transformation $(\tau, \bar{\tau}) \rightarrow\left(\chi^{\prime}, T\right)$ is a field redefinition which keeps the $\mathrm{SL}(2, \mathbb{R})$ invariance of the IIB supergravity action manifest, i.e. both $\tau=\chi+i e^{-\phi}$ and $\mathcal{T}=\chi^{\prime}+i T^{-1}$ appear in exactly the same way in the IIB supergravity action (compare eqs. (5.2) and (5.3) for $\operatorname{det} Q \rightarrow 0$ ). The reason is of course that the field redefinition in the $\operatorname{det} Q \rightarrow 0$ limit takes the form of an $\mathrm{SL}(2, \mathbb{R})$ transformation, eq. (5.19).

\subsection{What about $\operatorname{det} Q<0$ ?}

The $\mathrm{SL}(2, \mathbb{R})$ duality group has three subgroups: $\mathbb{R}, \mathrm{SO}(1,1)$ and $\mathrm{SO}(2)$. The transformations $e^{Q}$ with $\operatorname{det} Q=0, \operatorname{det} Q<0$ and $\operatorname{det} Q>0$ belong to these respective subgroups. In 
this subsection we will argue that there are no 7-branes which correspond to the $\mathrm{SO}(1,1)$ subgroup. ${ }^{8}$

The analogue of the field redefinition (5.4) for the case $\operatorname{det} Q<0$ is

$$
\frac{\tau-\tau_{0}^{+}}{\tau-\tau_{0}^{-}}=e^{2 \sqrt{-\operatorname{det} Q} \mathcal{T}} \quad \text { where } \quad \mathcal{T}=\chi^{\prime}+\frac{i}{2 \sqrt{-\operatorname{det} Q}} \operatorname{arccot} \frac{\mathrm{T}}{2 \sqrt{-\operatorname{det} Q}},
$$

where

$$
\tau_{0}^{ \pm}=-\frac{r}{2 q} \pm \frac{1}{q} \sqrt{-\operatorname{det} Q}
$$

which is such that $e^{Q} \tau_{0}^{ \pm}=\tau_{0}^{ \pm}$. The local form for $\tau$ whose monodromy around a point $z=0$ is of the form $\tau \rightarrow e^{Q} \tau$ with $\operatorname{det} Q<0$ is given by

$$
\left(\frac{\tau-\tau_{0}^{+}}{\tau-\tau_{0}^{-}}\right)^{\frac{i \pi}{\sqrt{-\operatorname{det} Q}}}=z .
$$

The problem with this possibility is that the local solution (5.22) as well as the conformal mapping (5.20) are ill defined at the points $\tau_{0}^{ \pm}$, i.e. the limit $\tau \rightarrow \tau_{0}^{ \pm}$does not exist. This means that the fixed points $\tau_{0}^{ \pm}$are not part of the IIB moduli space. Indeed when we consider the moduli space

$$
\frac{\operatorname{PSL}(2, \mathbb{R})}{\mathrm{SO}(2) \times \operatorname{PSL}(2, \mathbb{Z})}
$$

none of the orbifold points corresponds to $\tau_{0}^{ \pm}$.

\section{Towards the construction of the Q7-brane world-volume action}

\subsection{The Wess-Zumino term}

In previous sections we have discussed the coupling of the Q7-brane to the 8-form potential $q_{\alpha \beta} A_{8}^{\alpha \beta}$ and to its magnetically dual axion field $\chi^{\prime}$. In this section we shall construct the Wess-Zumino term which describes the coupling of the Q7-brane to all the gauge fields of IIB supergravity. As was argued in [15], in contrast to the D7-brane and its $\operatorname{SL}(2, \mathbb{R})$ partners (see eq. (2.1)), the invariance of the Q7-brane Wess-Zumino term under the gauge transformations (C.22) requires two Born-Infeld fields $A_{1}^{\alpha}(\alpha=1,2)$ on the Q7-brane worldvolume. Their field strengths are extended with the pullbacks of the doublet of the 2-forms $A_{2}^{\alpha}$ such that the generalized field strength

$$
\mathcal{F}_{2}^{\alpha}=d A_{1}^{\alpha}+A_{2}^{\alpha}=F_{2}^{\alpha}+A_{2}^{\alpha}
$$

is invariant under the gauge transformations

$$
\delta A_{2}^{\alpha}=d \phi_{1}, \quad \delta A_{1}=-\phi_{1}+d \phi_{0},
$$

\footnotetext{
${ }^{8}$ The nonexistence of a 7 -brane with $\operatorname{det} Q<0$ has consequences for the vacuum structure of $\operatorname{SO}(1,1)$ gauged supergravities in 9 dimensions. Certain gauged 9-dimensional supergravities can be obtained by performing a Schwerk-Schwarz reduction in which one gauges a subgroup of $\operatorname{SL}(2, \mathbb{R})$ 2, 4 . This corresponds to performing a reduction with 7-branes in the background. The fact that there is no well-defined 7-brane for $\operatorname{det} Q<0$ means that the $\mathrm{SO}(1,1)$ 9-dimensional gauged supergravity has no well-defined domain-wall vacuum.
} 
where $\phi_{1}$ and $\phi_{0}$ are world-volume 1-form and 0-form gauge transformation parameters, respectively.

We demand that the Q7-brane Wess-Zumino term satisfies the following three conditions:

1. it is invariant (up to a total derivative), under the gauge transformations (C.22),

2. it is monodromy neutral (i.e. invariant under the $\operatorname{SL}(2, \mathbb{R})$ transformation $e^{Q}$ in which $Q$ contains the charges of the 7-brane) and

3. it reduces in the $\operatorname{det} Q \rightarrow 0$ limit to the $(p, q) 7$-brane Wess-Zumino term of [15] with a single BI field.

The condition that the 7-brane world-volume action must be monodromy neutral follows from the fact that the 7-branes are always located at fixed points of the monodromy $\tau \rightarrow e^{Q} \tau$. In terms of the scalars $T$ and $\chi^{\prime}$ this means that the action must be invariant under the shift symmetry $\chi^{\prime} \rightarrow \chi^{\prime}+1$ ( $T$ is monodromy neutral). The WZ term satisfying the above requirements has the following form (where for simplicity we skip the wedge product symbol)

$$
\begin{aligned}
L_{\mathrm{WZ}}= & -q_{\alpha \beta}\left[A_{8}^{\alpha \beta}+\frac{1}{16} A_{6}^{(\alpha} A_{2}^{\beta)}+\frac{1}{12} A_{4} A_{2}^{\alpha} A_{2}^{\beta}-\left(\frac{1}{4} A_{6}^{(\alpha}-\frac{1}{3} A_{4} A_{2}^{(\alpha)}\right) \mathcal{F}_{2}^{\beta)}+\frac{1}{2} A_{4} \mathcal{F}_{2}^{\alpha} \mathcal{F}_{2}^{\beta}\right. \\
& \left.+\left(\frac{1}{12} A_{2}^{\alpha} A_{2}^{\beta}-\frac{1}{4} A_{2}^{(\alpha} \mathcal{F}_{2}^{\beta)}+\frac{1}{4} \mathcal{F}_{2}^{\alpha} \mathcal{F}_{2}^{\beta}\right) \frac{i}{16} \epsilon_{\gamma \delta} A_{2}^{\gamma} \mathcal{F}_{2}^{\delta}\right]+a(T) q_{\alpha \beta} \mathcal{F}_{2}^{\alpha} \mathcal{F}_{2}^{\beta} \mathcal{F}_{2}^{\gamma} \mathcal{F}_{2}^{\delta} q_{\gamma \delta} \\
& +\frac{1}{6 \cdot 8^{3}}(\operatorname{det} Q)^{1 / 2}\left[b(T) e^{-4 i \operatorname{sign}(q) \sqrt{\operatorname{det} Q} \chi^{\prime}} \frac{i}{\left(\operatorname{Im} \tau_{0}\right)^{2}} \times\right. \\
& \left.\times\left(-i\left(\mathcal{F}_{2}^{1}-\mathcal{F}_{2}^{2}\right)+\tau_{0}\left(\mathcal{F}_{2}^{1}+\mathcal{F}_{2}^{2}\right)\right)^{4}+\text { c.c. }\right],
\end{aligned}
$$

where $a(T)$ and $b(T)$ are undetermined real and complex-valued functions of $T$, respectively. The first term (in the square brackets) is completely fixed by the requirement of gauge invariance. In order for the WZ term (6.2) to reduce to the $(p, q)$ 7-brane Wess-Zumino term of [15] we must have that $a(T) \rightarrow$ constant and $b(T) \rightarrow 1$ when $\operatorname{det} Q \rightarrow 0$. It is expected that the form of the functions $a(T)$ and $b(T)$ will be fixed by world-volume supersymmetry. The last term in (6.2) describes the coupling of the Q7-brane Born-Infeld fields to the axion $\chi^{\prime}$. This term is invariant by itself under the shift symmetry $\chi^{\prime} \rightarrow \chi^{\prime}+1$ since the generalized BI field strengths are combined into the 'eigenform' of the operator $e^{Q}$ in a way similar to the RR and NSNS 2-forms of eq. (5.12), namely

$$
\begin{aligned}
e^{Q}: \frac{i}{\left(\operatorname{Im} \tau_{0}\right)^{1 / 2}}\left(i\left(\mathcal{F}_{2}^{1}-\mathcal{F}_{2}^{2}\right)-\tau_{0}\left(\mathcal{F}_{2}^{1}+\mathcal{F}_{2}^{2}\right)\right) \rightarrow \\
\rightarrow e^{i \operatorname{sign}(q) \sqrt{\operatorname{det} Q}} \frac{i}{\left(\operatorname{Im} \tau_{0}\right)^{1 / 2}}\left(i\left(\mathcal{F}_{2}^{1}-\mathcal{F}_{2}^{2}\right)-\tau_{0}\left(\mathcal{F}_{2}^{1}+\mathcal{F}_{2}^{2}\right)\right) .
\end{aligned}
$$

Though, in the form presented in eq. (6.2) the term containing the axion $\chi^{\prime}$ is not manifestly $\mathrm{SU}(1,1)$ covariant, it can be rewritten in an $\mathrm{SU}(1,1)$ covariant manner, i.e. with the scalars appearing via $V_{ \pm}^{1,2}$. However, in the $\mathrm{SU}(1,1)$ covariant form the role played by $\chi^{\prime}$ is no 
longer manifest and the resulting expression is more complicated. So we do not present it here.

\subsection{The Dirac-Born-nfeld part of the Q7-brane action}

To obtain the form of the DBI part of the Q7-brane action we perform a supersymmetry variation of the IIB background fields which appear in the WZ term, with the parameters corresponding to the supersymmetries which are not broken by the 7-brane and hence leave the action invariant (for a detailed generic discussion of this point see [17]). The terms obtained by performing a supersymmetry variation of the WZ term must combine with terms which result from a supersymmetry variation of the DBI part of the action to yield the 7-brane supersymmetry projector. It can be shown that up to terms which are cubic or quartic in the BI field strength $\mathcal{F}_{2}^{\alpha}$ the supersymmetry variation of the WZ term (6.2) is given by

$$
\begin{gathered}
\delta_{\epsilon} S_{\mathrm{WZ}}=-\int_{\mathcal{M}_{8}} d^{8} \xi\left(\sqrt { - g _ { ( 8 ) } } q _ { \alpha \beta } \left[V_{-}^{\alpha} V_{-}^{\beta} \bar{\epsilon}_{C} i \gamma_{\underline{8}} \gamma_{\underline{9}} \lambda+V_{+}^{\alpha} V_{-}^{\beta} \bar{\epsilon} \gamma_{\underline{8}} \gamma_{\underline{9}} \gamma^{\mu} \Psi_{\mu}+\frac{1}{8}\left(V_{-}^{\alpha} \bar{\epsilon} i \gamma_{\underline{8}} \gamma_{\underline{9}} \gamma_{\mu \nu} \lambda\right.\right.\right. \\
\left.+4 i V_{-}^{\alpha} \bar{\epsilon}_{C} i \gamma_{\underline{8}} \gamma_{\underline{9}} \gamma_{[\mu} \Psi_{\nu]}\right) \mathcal{F}^{\beta \mu \nu}+\frac{1}{4} V_{-}^{\alpha} \bar{\epsilon}_{C} \gamma_{\underline{8}} \gamma_{\underline{9}} \gamma^{\mu \nu} \gamma^{\rho} \Psi_{\rho} \mathcal{F}_{\mu \nu}^{\beta} \\
\left.\left.+\frac{1}{32} \bar{\epsilon} \gamma_{\underline{8}} \gamma_{\underline{9}} \gamma^{\rho \mu \nu \sigma \tau} \Psi_{\rho} \mathcal{F}_{\mu \nu}^{\alpha} \mathcal{F}_{\sigma \tau}^{\beta}\right]+ \text { c.c. }\right)
\end{gathered}
$$

where $\lambda$ and $\Psi_{\mu}$ are (the pullbacks of) the dilatino and gravitino field, respectively. The supersymmetry transformations of the IIB fields can be found in 18]. Greek indices refer to world-volume indices and Latin indices to target space indices. The underlined labels $\underline{8}$ and $\underline{9}$ denote flat tangent space indices. To perform the supersymmetry variation, the following gamma matrix duality was used

$$
-\epsilon^{\mu_{1} \ldots \mu_{8}} \gamma_{\mu_{1} \ldots \mu_{k}}=(-1)^{k(k-1) / 2} k ! \gamma^{\mu_{k+1} \ldots \mu_{8}} \gamma_{\underline{0}} \gamma_{\underline{1}} \ldots \gamma_{\underline{I}} \sqrt{-g_{(8)}},
$$

where $\epsilon^{\mu_{1} \ldots \mu_{8}}$ is the $8 \mathrm{D}$ Levi-Cività symbol. Further, in static gauge we have

$$
\begin{aligned}
& \gamma_{\underline{0}} \gamma_{\underline{1}} \cdots \gamma_{\underline{\underline{\underline{T}}}} \lambda=\gamma_{\underline{8}} \gamma_{\underline{\underline{9}}} \lambda, \\
& \gamma_{\underline{0}} \gamma_{\underline{1}} \cdots \gamma_{\underline{\underline{\gamma}}} \Psi_{\mu}=-\gamma_{\underline{8}} \gamma_{\underline{\underline{9}}} \Psi_{\mu}
\end{aligned}
$$

which follows from the chirality properties $\gamma_{11} \lambda=\lambda$ and $\gamma_{11} \Psi_{i}=-\Psi_{i}$. Other useful identities are the Clebsch-Gordon decompositions

$$
\begin{aligned}
\gamma_{\rho \mu \nu} & =\gamma_{\mu \nu} \gamma_{\rho}+2 g_{\rho[\mu} \gamma_{\nu]}, \\
\gamma_{\rho \mu \nu \sigma \tau} & =\gamma_{\mu \nu \sigma \tau} \gamma_{\rho}+4 g_{\rho[\mu} \gamma_{\nu \sigma \tau]}, \\
\gamma_{\mu \nu \sigma \tau} & =\gamma_{\mu \nu} \gamma_{\sigma \tau}+2 g_{\mu[\sigma} g_{\tau] \nu}-2 g_{\mu[\sigma} \gamma_{\tau] \nu}+2 g_{\nu[\tau} \gamma_{\sigma] \mu} .
\end{aligned}
$$

If we take for the first few terms of the DBI part of the Q7-brane action the following one

$$
S_{\mathrm{DBI}}=-\int_{\mathcal{M}_{8}} d^{8} \xi \sqrt{-g_{(8)}}\left(T+\frac{1}{4} q_{\alpha \beta} \mathcal{F}_{\mu \nu}^{\alpha} \mathcal{F}^{\beta \mu \nu}+\ldots\right)
$$


then for each term appearing in $\delta_{\epsilon} L_{\mathrm{DBI}}$ there exists a corresponding term in $\delta_{\epsilon} L_{\mathrm{WZ}}$ such that they make up the projector

$$
P=\frac{1}{2}\left(1+i \gamma_{\underline{8}} \gamma_{\underline{9}}\right)
$$

Hence, the supersymmetries which are not broken by the 7-brane are those for which $P \epsilon=0$. The last two terms in the square brackets of (6.4) are not canceled by terms in (6.11). These terms require the modification of the projector $P$ by terms which include $\mathcal{F}_{2}$, analogous to those which appear in the kappa-symmetry projector of the D7-brane. The above calculation generalizes the one of [12] up to terms which are second order in the BI field strength. We conclude that both Born-Infeld vectors carry propagating degrees of freedom. In the next section we shall briefly discuss the possibility of reducing the number of the Born-Infeld degrees of freedom by imposing a duality relation between their field strengths.

\section{Discussion}

In this paper we have considered the coupling of the Q7-brane to the bosonic sector of IIB supergravity and the structure of its world-volume action. In the static brane limit, in particular when there are no BI fields on the 7-brane, the coupling is best described using a new basis of the scalar manifold in which the fields are $T$ and $\chi^{\prime}$. The field $T$ is associated with the tension of the Q7-brane whereas $\chi^{\prime}$ is the axion dual to $q_{\alpha \beta} A_{8}^{\alpha \beta}$, the 8-form to which the Q7-brane couples electrically. Extending the construction by coupling the Q7-brane to 0-, 2-, 4- and 6-form fields described by the gauge invariant Wess-Zumino term requires the use of two Born-Infeld gauge fields. At present the microscopic origin of these two BI fields and of the Q7-brane itself is unclear. Below we only present some speculations regarding these issues.

Since the Q7-branes preserve half of IIB $\mathrm{D}=10$ supersymmetry their $d=8$ worldvolume theory is expected to possess 16 supersymmetries and to have an equal number of bosonic and fermionic physical degrees of freedom. In the case of the $(p, q) 7$-branes there is a single Born-Infeld field which in $d=8$ has 6 degrees of freedom, two transverse scalars and 8 on-shell Goldstone fermion modes so the number of the bosonic and fermionic degrees of freedom match. We do not know the full bosonic Q7-brane action but the part that we do know has in 8 -dimensions a number of $14=6+6+2$ bosonic degrees of freedom (two vectors and 2 (embedding) scalars) while there are only 8 fermionic physical degrees of freedom associated with the Goldstone fermions of $1 / 2$ bulk supersymmetry spontaneously broken by the Q7-brane.

It may happen that, as in the case of the duality-symmetric formulation of the D3brane [19, 20], the two Born-Infeld fields are actually not independent but related to each other by a duality condition. If a duality relation between the two Born-Infeld fields which reduces by half their degrees of freedom does take place, the number of bosonic and fermionic degrees of freedom on the Q7-brane will match. A simple proposal for such a condition which reduces by half the number of BI physical modes, and which should 
probably be corrected by higher non-linear terms (like in the case of the M5-brane [21, 24] and the D3-brane 19, 201), looks as follows

$$
\mathcal{F}_{2}^{-} \mathcal{F}_{2}^{-}=i *\left(\mathcal{F}_{2}^{-} \mathcal{F}_{2}^{-}\right)
$$

(and the complex conjugate for $\mathcal{F}_{2}^{+}$) where the Hodge operation is taken in the 8dimensional worldvolume of the Q7-brane and $\mathcal{F}_{2}^{ \pm}=\mathcal{F}_{2}^{\alpha} V_{\mp}^{\beta} \epsilon_{\alpha \beta}$.

Another possibility is a duality relation which can be given in the schematic form ${ }^{9}$

$$
\mathcal{F}_{2}=*\left(\mathcal{F}_{2} \mathcal{F}_{2} \mathcal{F}_{2}\right)
$$

One can construct several relations of this kind by combining the Born-Infeld field strengths with the axion-dilaton matrix $V_{\mp}^{\alpha}$ and the Q7-brane charge $q_{\alpha \beta}$.

If there is no duality relation between the two BI fields then in order to have a balance in the number of physical degrees of freedom we need two extra scalars and 8 extra fermions. A possible explanation for the origin of these missing degrees of freedom might be the assumption that the Q7-brane is a bound state of two coincident $(p, q)$ 7-branes, one of which is $\mathrm{SL}(2, \mathbb{R})$ rotated with respect to the other, so that both e.g. the fundamental string and the D1-brane end on the Q7-brane. If this is indeed the case then the construction of the complete world-volume action for the Q7-brane can be, to some extent, analogous to the construction of the action for $\mathrm{N}$ coincident D-branes with non-Abelian Born-Infeld fields [27-29]. Note that the construction of the target space covariant and supersymmetric N D-brane action encountered serious problems which have not been completely solved (see e.g. 30-34 for different approaches to tackle these problems). Since in the case under consideration we deal with only two Abelian BI fields, one may hope that these problems can be easier overcome. There is no non-Abelian enhancement of the gauge symmetry since there are no open strings which connect two $(p, q)$ 7-branes whose $(p, q)$ charges differ.

Finally one could say that if gauge invariance requires two BI vectors then we should expect the excitations of a Q7-brane to be always in terms of a $(p, q)$ string and a $\left(p^{\prime}, q^{\prime}\right)$ string and not in terms of only one of them, which in turn suggests that the two $(p, q)$ and $\left(p^{\prime}, q^{\prime}\right)$ strings are in some relation to each other and may themselves also form a bound state. One could refer to such a bound state as a Q1-brane. It remains to be seen if Q1branes require a duality relation between the two BI vectors on the Q7-brane world-volume or not. Whether such Q1-branes exist and whether there might be other Qp-branes which naturally couple to the new axion-dilaton $\left(T, \chi^{\prime}\right)$ rather than to the conventional one is under study.

\section{Acknowledgments}

The authors are grateful to P. Argyres, I. Bandos, M. Cederwall, J. de Azcárraga, T. Ortín, P. Pasti and M. Tonin for useful and encouraging discussions. This work was partially supported by the EU MRTN-CT-2004-005104 grant in which E.B. is associated to Utrecht

\footnotetext{
${ }^{9}$ Duality relations similar to $(7.1)$ and $(7.2)$ were considered e.g. in 25, 26, for studying higher dimensional counterparts of instantonic solutions in Yang-Mills theory and their reduction to $\mathrm{D}=4$.
} 
University and D.S. to Padova University and by the INTAS Project 1000008-7928. J.H. was supported by a Breedte Strategie grant of the University of Groningen. D.S. also acknowledges support from the INFN Special Initiative TS11, the MIUR Contract PRIN2005023102 and the Ministerio de Educación y Ciencia and EU FEDER funds (FIS200502761), the Generalitat Valenciana, during his sabbatical stay in Valencia. J.H. wishes to thank the hospitality of the University of Padova.

\section{A. Conventions}

We use the mostly plus signature $-+\cdots+$. The Levi-Cività symbol is denoted by $\epsilon_{m_{1} \ldots m_{10}}$ where $\epsilon_{01 \cdots 9}=-\epsilon^{01 \cdots 9}=1$. We denote space-time indices by $m, n=0,1, \ldots, 9$ and 7 -brane world-volume indices by $\mu, \nu=0,1, \ldots, 7$. Underlined indices refer to flat tangent space indices. Target space-time fields and world-volume fields are denoted by the same symbols.

\section{B. Properties of the $\mathrm{SU}(1,1) / \mathrm{U}(1)$ scalar coset}

In this appendix we collect some basic facts about the $\mathrm{SU}(1,1) / \mathrm{U}(1)$ coset that will be needed in the main text.

The coset $\mathrm{SU}(1,1) / \mathrm{U}(1)$ consists of all $\mathrm{SU}(1,1)$ matrices $V$ which are identified under the transformations of the compact subgroup $\mathrm{U}(1)$. If one takes $V$ to depend on spacetime points $x$ then the equivalence under $\mathrm{U}(1)$ becomes a gauge symmetry. A (left-)coset representative $V$ transforms as

$$
V(x) \rightarrow g V(x) h(x)
$$

where $g \in \mathrm{SU}(1,1)$ and $h \in \mathrm{U}(1)$. We will parameterize the coset representative $V$ as

$$
V=\left(\begin{array}{cc}
V_{-}^{1} & V_{+}^{1} \\
V_{-}^{2} & V_{+}^{2}
\end{array}\right),
$$

where $\left(\bar{V}_{\mp}^{1}\right)=V_{ \pm}^{2}$ and $V_{-}^{\alpha} V_{+}^{\beta}-V_{-}^{\beta} V_{+}^{\alpha}=\epsilon^{\alpha \beta}$ with the $\mathrm{SU}(1,1)$ indices $\alpha, \beta=1,2$.

Using the matrix $V$ a left-invariant Lie algebra element of $\mathrm{SU}(1,1)$ can be written as

$$
V^{-1} \partial_{\mu} V=\left(\begin{array}{cc}
-i Q_{\mu} & P_{\mu} \\
\bar{P}_{\mu} & i Q_{\mu}
\end{array}\right),
$$

where $Q_{\mu}$ is real and transforms as a composite $\mathrm{U}(1)$ gauge field under local $\mathrm{U}(1)$ transformations. The fields $P$ and $Q$ are both invariant under global $\mathrm{SU}(1,1)$ transformations. In terms of the components of $V$ this equation reads

$$
\begin{aligned}
P_{\mu} & =-\epsilon_{\alpha \beta} V_{+}^{\alpha} \partial_{\mu} V_{+}^{\beta}, \\
\bar{P}_{\mu} & =\epsilon_{\alpha \beta} V_{-}^{\alpha} \partial_{\mu} V_{-}^{\beta}, \\
Q_{\mu} & =-i \epsilon_{\alpha \beta} V_{-}^{\alpha} \partial_{\mu} V_{+}^{\beta} .
\end{aligned}
$$


The gauge-covariant derivative of $P_{\mu}$ is defined in the standard way as $D_{\mu} P_{\nu}=$ $\left(\partial_{\mu}-2 i Q_{\mu}\right) P_{\nu}$. The Bianchi identity for $P_{\mu}$ is given by

$$
D_{[\mu} P_{\nu]}=0
$$

The $\mathrm{U}(1)$ gauge symmetry can be fixed by imposing the gauge $V_{-}^{1}=V_{+}^{2} \in \mathbb{R}$. In this gauge the matrix elements of $V$ can be parameterized by a complex scalar $\tau$ as

$$
\begin{aligned}
V_{-}^{1} & =V_{+}^{2}=\frac{|1-i \tau|}{2(\operatorname{Im} \tau)^{1 / 2}}, \\
V_{+}^{1} & =\bar{V}_{-}^{2}=\frac{1-i \bar{\tau}}{1+i \bar{\tau}} \frac{|1-i \tau|}{2(\operatorname{Im} \tau)^{1 / 2}} .
\end{aligned}
$$

Using this parametrization we have

$$
\begin{aligned}
P_{\mu} & =\frac{1}{\tau-\bar{\tau}} \frac{1-i \tau}{1+i \bar{\tau}} \partial_{\mu} \bar{\tau} \\
Q_{\mu} & =\frac{i}{2} \frac{1}{\tau-\bar{\tau}} \frac{1-i \bar{\tau}}{1-i \tau} \partial_{\mu} \tau+\frac{i}{2} \frac{1}{\tau-\bar{\tau}} \frac{1+i \tau}{1+i \bar{\tau}} \partial_{\mu} \bar{\tau}
\end{aligned}
$$

It is convenient to define the following gauge-invariant (right-invariant) matrix $p_{\mu}$ as follows

$$
p_{\mu}=V \mathcal{P}_{\mu} V^{-1}, \quad \text { with } \quad \mathcal{P}_{\mu}=\left(\begin{array}{cc}
0 & P_{\mu} \\
\bar{P}_{\mu} & 0
\end{array}\right)
$$

It can be shown that the components of $p_{\mu}$ are the three Noether currents which are associated to the global SU $(1,1)$ invariance of the scalar kinetic terms of the IIB Lagrangian. In terms of the matrix $V$ the matrix $\mathcal{P}_{\mu}$ is given by

$$
\mathcal{P}_{\mu}=\frac{1}{2}\left(V^{-1} \partial_{\mu} V+\left(V^{-1} \partial_{\mu} V\right)^{\dagger}\right)
$$

In terms of $p_{\mu}$ the Bianchi identity (B.7) can be written as

$$
\partial_{[\mu} p_{\nu]}-2 p_{[\mu} p_{\nu]}=0
$$

\section{IIB supergravity}

\section{C.1 Manifest SU $(1,1)$ covariant formulation}

In the conventions of [35] and [18] the bosonic part of the IIB supergravity action [14, 3] is given by

$$
\begin{aligned}
S= & \int_{\mathcal{M}_{10}}\left(* 1 R-2 \bar{P} \wedge * P-\frac{1}{2} \bar{G}_{3} \wedge * G_{3}-4 F_{5} \wedge * F_{5}+\frac{i}{2} F_{5} \wedge \epsilon_{\alpha \beta} A_{2}^{\alpha} \wedge F_{3}^{\beta}\right) \\
& -\int_{\mathcal{M}_{10}} d^{10} x \frac{1}{6 \partial_{r} a \partial^{r} a} \partial^{l} a(x) \mathcal{F}_{l m_{1} \ldots m_{4}} \mathcal{F}^{m_{1} \ldots m_{4} p} \partial_{p} a(x) .
\end{aligned}
$$


The forms $P$ (introduced in appendix B), $G_{3}$ and $F_{5}$ are defined via the following Bianchi identities

$$
\begin{aligned}
D P & =d P-2 i Q \wedge P=0, \\
D G_{3} & =d G_{3}-i Q \wedge G_{3}=-P \wedge \bar{G}_{3}, \\
d F_{5} & =-\frac{i}{8} G_{3} \wedge \bar{G}_{3} .
\end{aligned}
$$

The solution to the Bianchi identity for $G_{3}$ is given by $G_{3}=-\epsilon_{\alpha \beta} V_{+}^{\alpha} F_{3}^{\beta}$ where $F_{3}^{\beta}=$ $d A_{2}^{\beta}$. The 2 -forms, $A_{2}^{\alpha}$, transform as a doublet under $\mathrm{SU}(1,1)$ and transform under gauge transformations as $\delta A_{2}^{\alpha}=d \Lambda_{1}^{\alpha}$. The solution to the Bianchi identity for $F_{5}$ reads

$$
F_{5}=d A_{4}+\frac{i}{16} \epsilon_{\alpha \beta} A_{2}^{\alpha} \wedge F_{3}^{\beta}
$$

The last term in $($ C.1 $)$ containing $\mathcal{F}_{5} \equiv F_{5}-{ }^{*} F_{5}$ and the auxiliary scalar field $a(x)$ is the PST term. It ensures that on the mass shell the equations of motion of $A_{4}$ reduce to the self-duality condition on its field strength (see [14, 3] for details)

$$
\mathcal{F}_{5}=F_{5}-* F_{5}=0 \text {. }
$$

\section{C.2 Introducing 6-forms}

It is possible to dualize the 2 -forms, $A_{2}^{\alpha}$, to a doublet of 6 -forms $A_{6}^{\alpha}$ via the duality relation and Bianchi identity

$$
\begin{aligned}
& F_{7}^{\alpha}=i *\left(V_{-}^{\alpha} G_{3}-V_{+}^{\alpha} \bar{G}_{3}\right), \\
& d F_{7}^{\alpha}=4 F_{3}^{\alpha} \wedge F_{5} .
\end{aligned}
$$

We define

$$
G_{7}=-\epsilon_{\alpha \beta} V_{+}^{\alpha} F_{7}^{\beta}
$$

which satisfies the following Bianchi identity

$$
D G_{7}+P \wedge \bar{G}_{7}=4 G_{3} \wedge F_{5} .
$$

From equation (C.7) it follows that $G_{7}=i * G_{3}$.

We can write the NSD IIB action in the first order formalism as a function of $A_{2}^{\alpha}$ and $F_{7}^{\alpha}$ such that the variation with respect to $A_{2}^{\alpha}$ gives the Bianchi identity (C.8) and the variation with respect to $F_{7}^{\alpha}$ the duality relation (C.7). This is achieved by the following action

$$
\begin{aligned}
S=\int_{\mathcal{M}_{10}}(* 1 R-2 \bar{P} & \wedge * P-\frac{1}{2} \bar{G}_{7} \wedge * G_{7}-4 F_{5} \wedge * F_{5} \\
+ & \left.\frac{i}{2} F_{5} \wedge \epsilon_{\alpha \beta} A_{2}^{\alpha} \wedge F_{3}^{\beta}+\frac{i}{2} \epsilon_{\alpha \beta} A_{2}^{\alpha} \wedge d F_{7}^{\beta}\right) .
\end{aligned}
$$

The 2-forms $A_{2}^{\alpha}$ are auxiliary variables. Their equations of motion, the Bianchi identities (C.8), can be solved for $F_{7}^{\alpha}$ in terms of a doublet of 6 -form potentials $A_{6}^{\alpha}$, via

$$
F_{7}^{\alpha}=d A_{6}^{\alpha}+\frac{4}{3} A_{2}^{\alpha} \wedge F_{5}-\frac{8}{3} F_{3}^{\alpha} \wedge A_{4}
$$


One can substitute the on-shell value for $F_{7}^{\alpha}$ back into the action (C.11) obtaining an action for $A_{6}^{\alpha}$ in the second order formalism. If instead we substitute the $F_{7}^{\alpha}$ equation of motion back into the action we recover the action (C.1) (modulo the PST part).

\section{C.3 Introducing 8-forms}

We can introduce a triplet of 8 -forms, $A_{8}^{\alpha \beta}$, via the duality relation

$$
\begin{aligned}
& F_{9}^{\alpha \beta}=i *\left(V_{+}^{\alpha} V_{+}^{\beta} \bar{P}-V_{-}^{\alpha} V_{-}^{\beta} P\right) \\
& d F_{9}^{\alpha \beta}=\frac{1}{4} F_{3}^{(\alpha} \wedge F_{7}^{\beta)} .
\end{aligned}
$$

Solving the Bianchi identity (C.14) we find that $F_{9}^{\alpha \beta}$ in the $\mathrm{SU}(1,1)$ covariant formulation can be written as

$$
F_{9}^{\alpha \beta}=d A_{8}^{\alpha \beta}+\frac{1}{16} F_{7}^{(\alpha} \wedge A_{2}^{\beta)}-\frac{3}{16} F_{3}^{(\alpha} \wedge A_{6}^{\beta)} .
$$

$F_{9}^{\alpha \beta}$ satisfies the following $\mathrm{SU}(1,1)$ invariant constraint [3]

$$
\epsilon_{\alpha \gamma} \epsilon_{\beta \delta} V_{-}^{\alpha} V_{+}^{\beta} F_{9}^{\gamma \delta}=0 .
$$

The duality relation between the 8-forms and an axionic scalar follows from the duality relation (C.14) by contracting the latter with the $\mathrm{SU}(1,1)$ symmetric charge tensor $q_{\alpha \beta}$ introduced in section 3 and making use of equations (5.4) to (5.16)

$$
\frac{\operatorname{det} Q}{\left(\operatorname{Im} \tau_{0}\right)^{2}}\left|\left(\tau-\tau_{0}\right)\left(\tau-\bar{\tau}_{0}\right)\right|^{2} d \chi^{\prime}=\left(T^{2}-4 \operatorname{det} Q\right) d \chi^{\prime}=* q_{\alpha \beta} F_{9}^{\alpha \beta} .
$$

Eq. (C.17) makes manifest the statement that each 8-form is dual to an axionic scalar and that implementing this duality relation into the IIB action makes it mandatory to perform the field redefinition as given in eq. (5.4).

Modulo the PST term, the action for the bosonic sector of IIB supergravity which reproduces the duality relations between $F_{2}^{\alpha}$ and $F_{7}^{\alpha}$ and between $d \chi^{\prime}$ and $q_{\alpha \beta} F_{9}^{\alpha \beta}$ has the form

$$
\begin{aligned}
S= & \int_{\mathcal{M}_{10}}\left[* 1 R-\frac{1}{2} \frac{1}{T^{2}-4 \operatorname{det} Q}\left(d T \wedge * d T+q_{\alpha \beta} F_{9}^{\alpha \beta} \wedge * q_{\alpha \beta} F_{9}^{\alpha \beta}\right)-\frac{1}{2} \bar{G}_{7} \wedge * G_{7}\right. \\
& \left.-4 F_{5} \wedge * F_{5}+\frac{i}{2} F_{5} \wedge \epsilon_{\alpha \beta} A_{2}^{\alpha} \wedge F_{3}^{\beta}+\frac{i}{2} \epsilon_{\alpha \beta} A_{2}^{\alpha} \wedge d F_{7}^{\beta}+\chi^{\prime} d\left(q_{\alpha \beta} F_{9}^{\alpha \beta}\right)\right],
\end{aligned}
$$

where $F_{9}^{\alpha \beta}$ and $F_{7}^{\alpha}$ are considered as independent fields and $G_{7}$ depends on $F_{7}^{\alpha}$ and $\chi^{\prime}$ as given in eq. (C.9) in which $\phi$ and $\chi$ are expressed in terms of $T$ and $\chi^{\prime}$ using (5.4) and (3.26).

The form of (C.18) differs from the action (3.10). The former contains $F_{9}^{\alpha \beta}$ as an independent field, while the latter depends on $A_{8}^{\alpha \beta}$ and thus is more suitable for describing the minimal electric coupling of the Q7-brane. 


\section{C.4 Gauge field transformations}

In the $\mathrm{SU}(1,1)$ covariant formulation the $\mathrm{r}$-form gauge fields $(r=2,4,6,8)$ have the following gauge transformations:

$$
\begin{aligned}
& \delta A_{2}^{\alpha}=d \Lambda_{1}^{\alpha} \\
& \delta A_{4}=d \Lambda_{3}-\frac{i}{16} \epsilon_{\gamma \delta} \delta A_{2}^{\gamma} \wedge A_{2}^{\delta}, \\
& \delta A_{6}^{\alpha}=d \Lambda_{5}^{\alpha}+\frac{8}{3} A_{2}^{\alpha} \wedge \delta A_{4}-\frac{4}{3} A_{4} \wedge \delta A_{2}^{\alpha}+\frac{i}{12} A_{2}^{\alpha} \wedge \epsilon_{\gamma \delta} \delta A_{2}^{\gamma} \wedge A_{2}^{\delta}, \\
& \delta A_{8}^{\alpha \beta}=d \Lambda_{7}^{\alpha \beta}+\frac{3}{16} A_{2}^{(\alpha} \wedge \delta A_{6}^{\beta)}-\frac{1}{16} A_{6}^{(\alpha} \wedge \delta A_{2}^{\beta)}-\frac{1}{4} A_{2}^{\alpha} \wedge A_{2}^{\beta} \wedge \delta A_{4}+ \\
& \frac{1}{6} A_{4} \wedge A_{2}^{(\alpha} \wedge \delta A_{2}^{\beta)}-\frac{1}{12} A_{2}^{\alpha} \wedge A_{2}^{\beta} \wedge \frac{i}{16} \epsilon_{\gamma \delta} \delta A_{2}^{\gamma} \wedge A_{2}^{\delta} .
\end{aligned}
$$

\section{C.5 Manifest $\mathrm{SL}(2, \mathbb{R})$ covariant formulation}

We now formulate the IIB theory in the standard $(\tau, \bar{\tau})$ basis. The RR and NSNS 2-forms are denoted by $C_{2}$ and $B_{2}$, respectively. Their duals will be denoted by $C_{6}$ and $B_{6}$. They are defined by $B_{2,6}=\frac{1}{2}\left(A_{2,6}^{1}+A_{2,6}^{2}\right)$ and $C_{2,6}=\frac{i}{2}\left(A_{2,6}^{1}-A_{2,6}^{2}\right)$. The axion-dilaton field $\tau$ is $\tau=\chi+i e^{-\phi}$. The objects $P, Q, G_{3}, F_{5}$ and $G_{7}$ can be written $\operatorname{as}^{10}$

$$
\begin{aligned}
P & =\frac{d \bar{\tau}}{\tau-\bar{\tau}}, \\
Q & =i \frac{d(\tau+\bar{\tau})}{2(\tau-\bar{\tau})}, \\
G_{3} & =\frac{i}{(\operatorname{Im} \tau)^{1 / 2}}\left(-d C_{2}+\bar{\tau} d B_{2}\right) \\
F_{5} & =d A_{4}+\frac{1}{8}\left(C_{2} \wedge d B_{2}-B_{2} \wedge d C_{2}\right), \\
G_{7} & =\frac{i}{(\operatorname{Im} \tau)^{1 / 2}}\left(-d C_{6}+\bar{\tau} d B_{6}+\frac{4}{3}\left(-C_{2}+\bar{\tau} B_{2}\right) \wedge F_{5}\right. \\
& \left.\quad-\frac{8}{3}\left(-d C_{2}+\bar{\tau} d B_{2}\right) \wedge A_{4}\right) .
\end{aligned}
$$

In this formulation the following symmetry is manifest

$$
\tau \rightarrow \frac{a \tau+b}{c \tau+d} \quad \text { and } \quad\left(\begin{array}{c}
C_{2} \\
B_{2}
\end{array}\right) \rightarrow\left(\begin{array}{ll}
a & b \\
c & d
\end{array}\right)\left(\begin{array}{l}
C_{2} \\
B_{2}
\end{array}\right) \quad \text { with } \quad a d-b c=1 .
$$

\section{References}

[1] E.A. Bergshoeff, J. Hartong, T. Ortín and D. Roest, Seven-branes and supersymmetry, JHEP 02 (2007) 003 hep-th/0612072.

\footnotetext{
${ }^{10}$ To obtain these expressions one can start from the parametrization of $V_{ \pm}^{\alpha}$ given in $(\mathrm{B} .8)$ and the definitions of $P, Q$ and $G_{3}$. Then use the freedom to perform the local U(1) transformation $P \rightarrow e^{2 i \alpha} P$, $Q \rightarrow Q+d \alpha$ and $G_{3,7} \rightarrow e^{i \alpha} G_{3,7}$ with parameter $\alpha$ given by $e^{2 i \alpha}=\frac{1+i \bar{\tau}}{1-i \tau}$.
} 
[2] P. Meessen and T. Ortín, An $\mathrm{SL}(2, \mathbb{Z})$ multiplet of nine-dimensional type-II supergravity theories, Nucl. Phys. B 541 (1999) 195 hep-th/9806120.

[3] G. Dall'Agata, K. Lechner and M. Tonin, $D=10, N=I I B$ supergravity: Lorentz-invariant actions and duality, JHEP 07 (1998) 017 hep-th/9806140.

[4] E. Bergshoeff, U. Gran and D. Roest, Type IIB seven-brane solutions from nine-dimensional domain walls, Class. and Quant. Grav. 19 (2002) 4207 hep-th/0203202.

[5] P.A.M. Dirac, Quantised singularities in the electromagnetic field, Proc. Roy. Soc. Lond. A 133 (1931) 60; The theory of magnetic poles, Phys. Rev. 74 (1948) 817.

[6] S. Deser, A. Gomberoff, M. Henneaux and C. Teitelboim, Duality, self-duality, sources and charge quantization in abelian N-form theories, Phys. Lett. B 400 (1997) 80 hep-th/9702184.

[7] R. Medina and N. Berkovits, Pasti-Sorokin-Tonin actions in the presence of sources, Phys. Rev. D 56 (1997) 6388 hep-th/9704093.

[8] I.A. Bandos, N. Berkovits and D.P. Sorokin, Duality-symmetric eleven-dimensional supergravity and its coupling to M-branes, Nucl. Phys. B 522 (1998) 214 hep-th/9711055.

[9] M. Cederwall, A. von Gussich, B.E.W. Nilsson, P. Sundell and A. Westerberg, The Dirichlet super-p-branes in ten-dimensional type IIA and IIB supergravity, Nucl. Phys. B 490 (1997) 179 hep-th/9611159.

[10] B.R. Greene, A.D. Shapere, C. Vafa and S.-T. Yau, Stringy cosmic strings and noncompact Calabi-Yau manifolds, Nucl. Phys. B 337 (1990) 1.

[11] G.W. Gibbons, M.B. Green and M.J. Perry, Instantons and seven-branes in type IIB superstring theory, Phys. Lett. B 370 (1996) 37 hep-th/9511080.

[12] E.A. Bergshoeff, M. de Roo, S.F. Kerstan, T. Ortín and F. Riccioni, IIB nine-branes, JHEA 06 (2006) 006 hep-th/0601128.

[13] P. Pasti, D.P. Sorokin and M. Tonin, Duality symmetric actions with manifest space-time symmetries, Phys. Rev. D 52 (1995) 4277 hep-th/9506109; On Lorentz invariant actions for chiral p-forms, Phys. Rev. D 55 (1997) 6292 hep-th/9611100.

[14] G. Dall'Agata, K. Lechner and D.P. Sorokin, Covariant actions for the bosonic sector of $D=10$ IIB supergravity, Class. and Quant. Grav. 14 (1997) L195 hep-th/9707044.

[15] E.A. Bergshoeff, M. de Roo, S.F. Kerstan, T. Ortín and F. Riccioni, SL(2, R)-invariant IIB brane actions, JHEP 02 (2007) 007 hep-th/0611036.

[16] K. Lechner and P.A. Marchetti, Duality-invariant quantum field theories of charges and monopoles, Nucl. Phys. B 569 (2000) 529 [hep-th/9906079]; Interacting branes, dual branes and dyonic branes: a unifying lagrangian approach in d dimensions, JHEP 01 (2001) 003 [hep-th/0007076]; Chern kernels and anomaly cancellation in M-theory, Nucl. Phys. B 672 (2003) 264 hep-th/0302108;

K. Lechner, P.A. Marchetti and M. Tonin, Anomaly free effective action for the elementary m5-brane, Phys. Lett. B 524 (2002) 199 hep-th/0107061.

[17] I.A. Bandos, J.A. De Azcarraga and J.M. Izquierdo, Supergravity interacting with bosonic p-branes and local supersymmetry, Phys. Rev. D 65 (2002) 105010 hep-th/0112207;

I.A. Bandos, J.A. de Azcarraga, J.M. Izquierdo and J. Lukierski, On dynamical supergravity interacting with super-p-brane sources, hep-th/0211065. 
[18] E.A. Bergshoeff, M. de Roo, S.F. Kerstan and F. Riccioni, IIB supergravity revisited, JHEP 08 (2005) 098 hep-th/0506013.

[19] D. Berman, SL $(2, Z)$ duality of Born-Infeld theory from non-linear self-dual electrodynamics in 6 dimensions, Phys. Lett. B 409 (1997) 153 hep-th/9706208.

[20] A. Nurmagambetov, Duality-symmetric three-brane and its coupling to type IIB supergravity, Phys. Lett. B 436 (1998) 289 hep-th/9804157.

[21] P.S. Howe and E. Sezgin, $D=11, p=5$, Phys. Lett. B 394 (1997) 62 hep-th/9611008.

[22] E. Bergshoeff, R. Kallosh, T. Ortín, D. Roest and A. Van Proeyen, New formulations of $D=10$ supersymmetry and D8-O8 domain walls, Class. and Quant. Grav. 18 (2001) 3359 hep-th/0103233.

[23] A. Sen, F-theory and orientifolds, Nucl. Phys. B 475 (1996) 562 hep-th/9605150.

[24] M. Perry and J.H. Schwarz, Interacting chiral gauge fields in six dimensions and Born-Infeld theory, Nucl. Phys. B 489 (1997) 47 hep-th/9611065.

[25] D.H. Tchrakian, N-dimensional instantons and monopoles, J. Math. Phys. 21 (1980) 166 ; 't Hooft electromagnetic tensor for Higgs fields of arbitrary isospin, Phys. Lett. B 91 (1980) 415.

[26] F.A. Bais and P. Batenburg, Yang-Mills duality in higher dimensions, Nucl. Phys. B 269 (1986) 363 .

[27] A.A. Tseytlin, Born-Infeld action, supersymmetry and string theory, hep-th/9908105.

[28] W. Taylor and M. Van Raamsdonk, Multiple D0-branes in weakly curved backgrounds, Nucl. Phys. B 558 (1999) 63 hep-th/9904095; Multiple Dp-branes in weak background fields, Nucl. Phys. B 573 (2000) 703 hep-th/9910052.

[29] R.C. Myers, Dielectric-branes, JHEP 12 (1999) 022 hep-th/9910053.

[30] E.A. Bergshoeff, M. de Roo and A. Sevrin, Non-abelian Born-Infeld and $\kappa$-symmetry, 囚. Math. Phys. 42 (2001) 2872 hep-th/0011018;

E.A. Bergshoeff, A. Bilal, M. de Roo and A. Sevrin, Supersymmetric non-abelian Born-Infeld revisited, JHEP 07 (2001) 029 hep-th/0105274.

[31] D.P. Sorokin, Coincident (super)-Dp-branes of codimension one, JHEP 08 (2001) 022 hep-th/0106212;

S. Panda and D. Sorokin, Supersymmetric and kappa-invariant coincident D0-branes, JHEP 02 (2003) 055 hep-th/0301065.

[32] J.M. Drummond, P.S. Howe and U. Lindström, Kappa-symmetric non-abelian Born-Infeld actions in three dimensions, Class. and Quant. Grav. 19 (2002) 6477 [hep-th/0206148].

[33] S.F. Hassan, $N=1$ worldsheet boundary couplings and covariance of non-abelian worldvolume theory, hep-th/0308201.

[34] P.S. Howe, U. Lindström and L. Wulff, Superstrings with boundary fermions, JHEP 08 (2005) 041 hep-th/0505067]; On the covariance of the Dirac-Born-Infeld-Myers action, JHEP 02 (2007) 070 hep-th/0607156; ; $\kappa$-symmetry for coincident D-branes, JHEP 09 (2007) 010 arXiv:0706.2494.

[35] J.H. Schwarz, Covariant field equations of chiral $N=2 D=10$ supergravity, Nucl. Phys. B 226 (1983) 269. 